\title{
1 Intimate genetic relationships and fungicide resistance 2 in multiple strains of human pathogenic fungus 3 Aspergillus fumigatus isolated from a plant bulb
}

5 Hiroki Takahashi ${ }^{1,2,3}$, Sayoko Oiki ${ }^{4}$, Yoko Kusuya ${ }^{1}$, Syun-ichi Urayama ${ }^{4,5}$,

6 Daisuke Hagiwara ${ }^{4,5, *}$

7

$8{ }^{1}$ Medical Mycology Research Center, Chiba University, 1-8-1 Inohana, Chuo-ku, Chiba

9 260-8673, Japan

$10{ }^{2}$ Molecular Chirality Research Center, Chiba University, 1-33 Yayoi-cho, Inage-ku,

11 Chiba 263-8522, Japan

$12{ }^{3}$ Plant Molecular Science Center, Chiba University, 1-8-1 Inohana, Chuo-ku, Chiba

13 260-8675, Japan

$14{ }^{4}$ Faculty of Life and Environmental Sciences, University of Tsukuba, 1-1-1 Tennodai,

15 Tsukuba, Ibaraki 305-8577, Japan

$16{ }^{5}$ Microbiology Research Center for Sustainability, University of Tsukuba, 1-1-1

17 Tennodai, Tsukuba, Ibaraki 305-8577, Japan

19 * Corresponding author: D. Hagiwara

20 hagiwara.daisuke.gb@u.tsukuba.ac.jp

22 Running title

23 Plant bulb-derived azole resistant $A$. fumigatus

25 ORCIDs

26 D. Hagiwara: 0000-0003-1382-3914

27 H. Takahashi: 0000-0001-5627-1035 


\section{Summary}

30 Fungal infections are increasingly dangerous because of

31 environmentally-dispersed resistance to antifungal drugs. Azoles are commonly

32 used antifungal drugs, but they are also used as fungicides in agriculture, which

33 may enable enrichment of azole-resistant strains of the human pathogen

34 Aspergillus fumigatus in the environment. Understanding of environmental

35 dissemination and enrichment of genetic variation associated with azole

36 resistance in $A$. fumigatus is required to suppress resistant strains. Here, we

37 focused on eight strains of azole-resistant $A$. fumigatus isolated from a single

38 tulip bulb for sale in Japan. This set includes strains with

$39 \mathrm{TR}_{34} / \mathrm{L} 98 \mathrm{H} / \mathrm{T} 289 \mathrm{~A} / \mathrm{I364V} / \mathrm{G} 448 \mathrm{~S}$ and $\mathrm{TR}_{46} / \mathrm{Y} 121 \mathrm{~F} / \mathrm{T} 289 \mathrm{~A} / \mathrm{S} 363 \mathrm{P} / \mathrm{I364V} / \mathrm{G} 448 \mathrm{~S}$

40 mutations in the cyp51A gene, which showed higher tolerance to several azoles

41 than strains harboring $\mathrm{TR}_{46} / \mathrm{Y} 121 \mathrm{~F} / \mathrm{T} 289 \mathrm{~A}$ mutation. The strains were typed by

42 microsatellite typing, single nucleotide polymorphism profiles, and mitochondrial

43 and nuclear genome analyses. The strains grouped differently using each typing

44 method, suggesting historical genetic recombination among the strains. Our

45 data also revealed that some strains isolated from the tulip bulb showed

46 tolerance to other classes of fungicide, such as Qol and carbendazim, followed

47 by related amino acid alterations in the target proteins. Considering

48 spatial-temporal factors, plant bulbs are an excellent environmental niche for

49 fungal strains to encounter partners, and to obtain and spread

50 resistance-associated mutations. 


\section{Introduction}

53 Azoles are versatile compounds that show outstanding activity against a wide

54 range of fungi, including plant and human pathogens. These compounds play an

55 essential role in agricultural and clinical settings as fungicides and antifungal

56 drugs (Fisher et al, 2018, Price et al, 2015). Their main mode of action is

57 inhibition of the ergosterol biosynthesis pathway by inhibiting Cyp51, which

58 functions as an 14-alpha-demethylase critical for the biosynthesis. Azole

59 fungicides, known as demethylase inhibitors (DMIs), include triazole and

60 imidazole compounds such as tebuconazole, propiconazole, triflumizole, and

61 prochloraz. They are widely used to protect crops and fruits against pathogens

62 by application during cultivation and postharvest preservation, as well as for

63 seed disinfection. In medicine, azole drugs are essential options to combat

64 dermatophytes and deep-seated fungal pathogens, such as Trichophyton

65 rubrum and Aspergillus fumigatus, respectively. Azoles are the only class of

66 compound used to control fungi in both agriculture and medicine.

67 A. fumigatus is a major causative agent of aspergillosis and ubiquitously

68 present in the environment as a saprobe. A limited number of antifungals are

69 approved for therapy of $A$. fumigatus infection; voriconazole (VRCZ) and

70 itraconazole (ITCZ) are the first-line drugs for the treatment of pulmonary

71 infection (Jenks and Hoenigl, 2018). However, this antifungal therapy is

72 threatened by azole-resistant $A$. fumigatus, strains of which have been

73 increasingly isolated since the beginning of this century (Howard et al, 2009).

74 The resistance mechanisms to azole drugs that have been identified in $A$.

75 fumigatus from clinical settings are mutations in Cyp51A, HMG-CoA reductase

76 HMG1, and a subunit of CCAAT-binding complex HapE, and overexpression of

77 cdr1B, which encodes an ABC transporter (Hagiwara et al, 2016a, Nywening et

78 al, 2020, Hagiwara et al, 2018, Rybak et al, 2019, Camps et al, 2012,

79 Hortschansky, et al, 2020, Fraczek et al, 2013). These azole resistance 
80 mutations are thought to have emerged during therapy with prolonged azole

81 treatment.

82 However, in addition to treatment-based resistance, environmentally derived

83 resistance has been considered as a non-negligible source of azole drug

84 resistance of $\boldsymbol{A}$. fumigatus during the last decade (Berger et al, 2017, Lestrade et

85 al, 2019). Typical resistant strains from the environment carry a tandem repeat

86 (TR) and single-nucleotide polymorphisms (SNPs) in the promoter and coding

87 regions of the cyp51A gene, respectively. The most prevalent variants are

$88 \mathrm{TR}_{34} / \mathrm{L} 98 \mathrm{H}$ and $\mathrm{TR}_{46} / \mathrm{Y} 121 \mathrm{~F} / \mathrm{T} 289 \mathrm{~A}$, which were isolated for the first time from

89 patients in Europe in 1998 and North America in 2008, respectively (Jeanvoine

90 et al, 2020). The mutants with $\mathrm{TR}_{34}$ typically show high resistance to ITCZ,

91 whereas the strains with $\mathrm{TR}_{46}$ show VRCZ resistance, but some are

92 pan-azole-resistant strains. These genotypes were later recovered from

93 environments worldwide (Schoustra et al, 2019, Resendiz et al, 2018, Hagiwara,

94 2018). Diverse resistant mutants with tandem repeats in the Cyp51A-encoding

95 gene have been reported (Table 1).

96

97 Table 1. Reported Cyp51A variants with tandem repeats in azole-resistant $A$.

98 fumigatus

\begin{tabular}{|l|l|l|}
\hline Cyp51A allele & Country & References \\
\hline $\mathrm{TR}_{34} / \mathrm{L} 98 \mathrm{H}$ & Many places & - \\
\hline $\mathrm{TR}_{46} / \mathrm{Y} 121 \mathrm{~F} / \mathrm{T} 289 \mathrm{~A}$ & Many places & - \\
\hline $\mathrm{TR}_{53}$ & Colombia & Alvarez-Moreno et al, 2017 \\
\hline $\mathrm{TR}_{34} / \mathrm{L} 98 \mathrm{H} / \mathrm{S} 302 \mathrm{~N}$ & The Netherlands & Schoustra et al, 2019 \\
\hline $\mathrm{TR}_{34} / \mathrm{L} 98 \mathrm{H} / \mathrm{F} 495 \mathrm{I}$ & The Netherlands & Schoustra et al, 2019 \\
\hline $\mathrm{TR}_{34} / \mathrm{L} 98 \mathrm{H} / \mathrm{L} 343 \mathrm{H}$ & The Netherlands & Schoustra et al, 2019 \\
\hline $\mathrm{TR}_{34} / \mathrm{L} 98 \mathrm{H} / \mathrm{E} 356 \mathrm{~V}$ & The Netherlands & Schoustra et al, 2019 \\
\hline $\mathrm{TR}_{34} / \mathrm{L} 98 \mathrm{H} / \mathrm{S} 297 \mathrm{~T} / \mathrm{F} 495 \mathrm{I}$ & The Netherlands & Schoustra et al, 2019, Cao et al, 2020 \\
\hline
\end{tabular}




\begin{tabular}{|c|c|c|}
\hline $\mathrm{TR}_{34} / \mathrm{L} 98 \mathrm{H} / \mathrm{T} 289 \mathrm{~A} / \mathrm{I364V} / \mathrm{G} 448 \mathrm{~S}$ & Japan & Nakano et al, 2020, this study \\
\hline $\mathrm{TR}_{46} / \mathrm{Y} 121 \mathrm{~F} / \mathrm{T} 289 \mathrm{~A} / \mathrm{I364V}$ & The Netherlands & Schoustra et al, 2019 \\
\hline $\mathrm{TR}_{46} / \mathrm{Y} 121 \mathrm{~F} / \mathrm{M} 172 \mathrm{I} / \mathrm{T} 289 \mathrm{~A} / \mathrm{G} 448 \mathrm{~S}$ & $\begin{array}{l}\text { The Netherlands, Japan, } \\
\text { Iran }\end{array}$ & $\begin{array}{l}\text { Zhang et al, 2017, Nakano et al, 2020, } \\
\text { Ahangatkani et al, 2020, Fraaije et al, } 2020\end{array}$ \\
\hline $\mathrm{TR}_{46} / \mathrm{Y} 121 \mathrm{~F} / \mathrm{T} 289 \mathrm{~A} / \mathrm{S} 363 \mathrm{P} / \mathrm{I364V/G448S}$ & The Netherlands, Japan & $\begin{array}{l}\text { Nakano et al, 2020, Fraaije et al, } 2020 \text {, } \\
\text { Zhang et al, 2021, this study }\end{array}$ \\
\hline $\mathrm{TR}^{3}{ }_{46} / \mathrm{Y} 121 \mathrm{~F} / \mathrm{M} 172 \mathrm{I} / \mathrm{T} 289 \mathrm{~A} / \mathrm{G} 448 \mathrm{~S}$ & The Netherlands, Japan & Zhang et al, 2017, Nakano et al, 2020 \\
\hline $\mathrm{TR}_{46}^{4} / \mathrm{Y} 121 \mathrm{~F} / \mathrm{M} 172 \mathrm{I} / \mathrm{T} 289 \mathrm{~A} / \mathrm{G} 448 \mathrm{~S}$ & The Netherlands & Zhang et al, 2021 \\
\hline $\mathrm{TR}_{92} / \mathrm{Y} 121 \mathrm{~F} / \mathrm{M} 172 \mathrm{I} / \mathrm{T} 289 \mathrm{~A} / \mathrm{G} 448 \mathrm{~S}$ & The Netherlands & Zhang et al, 2021 \\
\hline
\end{tabular}

99

100

101

102

103

104

105

106

107

108

109

110

111

112

113

114

115

116

117

Recently, a possible environmental hot spot for azole-resistant $A$. fumigatus was proposed (Zhang et al, 2017). The TR-type mutants were prevalently isolated from agricultural compost containing azole fungicide residues, whereas azole-free compost was dominated by wild-type (WT) $A$. fumigatus. This view was also supported in other studies (Schoustra et al, 2019, Zhang et al, 2021), indicating that azole-resistant strains are enriched under the selective pressure of environmental azoles. The work by Zhang et al. also suggested that sexual reproduction plays an important role in developing and evolving new cyp51A alleles for drug resistance in compost (Zhang et al, 2017). Taking into consideration that TR-type drug-resistant $A$. fumigatus mutants show cross-resistance to DMIs (Snelders et al., 2012), azole-containing environmental niches may serve as evolutionary incubators through genetic recombination.

The propagation of azole-resistant $A$. fumigatus has been studied in an epidemiological manner using microsatellite analysis by short tandem repeats for $A$. fumigatus (STRAf), which is a widely accepted intraspecies typing method with high-resolution discriminatory power (de Valk et al, 2005). TR-type mutant strains were spread worldwide. Some isolates from multiple countries were genetically closely related to each other and some had identical microsatellite 
118 patterns (Pontes et al, 2020, Cao et al, 2020, Wang et al, 2018, Hagiwara et al,

119 2016b). Besides such international propagation, intranational clonal expansion

120 was also reported in several countries (Ahangarkani et al, 2020, Chowdhary et al,

121 2012). Recent population genomic studies revealed that the azole-resistant

122 strains are globally distributed. The isolates were divided into two broad clades,

123 and TR mutants belong to the populations in an uneven manner (Sewell et al,

124 2019). These data suggest that azole resistance primarily expanded by asexual

125 and sexual propagation from a limited number of ancestors with TR-type

126 mutation, rather than locally and independently emerging in each environment.

127 It was recently proposed that resistant $A$. fumigatus strains are transferred

128 internationally via imported plant bulbs (Dunne et al, 2017). Plant bulbs

129 produced in the Netherlands and sold in Ireland were contaminated with TR-type

130 A. fumigatus mutants. Similar cases were also reported by two independent

131 Japanese groups (Hagiwara 2020, Nakano et al, 2020); azole-resistant $A$.

132 fumigatus with diverse Cyp51A variants were isolated from plant bulbs that were

133 imported from the Netherlands and sold in Japanese gardening shops. These

134 studies suggest that the wide spread of azole-resistant $A$. fumigatus mutants is

135 attributable in part to trade in agricultural products including plant bulbs.

136 In the present study, to further understand genetic variations in plant

137 bulb-associated isolates, we focused on eight $A$. fumigatus strains that were

138 co-isolated from a single tulip bulb in a previous screening study (Hagiwara,

139 2020). Sensitivity to medical and agricultural azoles, as well as other classes of

140 fungicides, was compared between the strains. Whole genome comparison of

141 the eight strains showed several fragmental overlaps of their genomes,

142 suggesting genetic recombination had occurred between strains in the single

143 bulb. Our work indicates that plant bulbs are not only a vehicle for the pathogen

144 but also a place where the pathogen can evolve its drug resistance. 


\section{Results}

\section{Variation of Cyp51A mutation in strains from a single bulb}

148 In a previous study, eight strains of $A$. fumigatus were isolated from a single tulip

149 bulb as different colonies (hereafter referred to as strains 3-1-A to 3-1-H)

150 (Hagiwara, 2020). Strain 3-1-H has no TR or SNPs in cyp51A, whereas $\mathrm{TR}_{34}$ or

$151 \mathrm{TR}_{46}$ occur in combination with various SNPs in the other seven strains (Table 2).

152 Strains 3-1-A, 3-1-E, 3-1-F, and 3-1-G have a typical variant, TR46/Y121F/T289A.

153 Strain 3-1-D has mutations S363P, I364V, and G448S as well as

$154 \mathrm{TR}_{46} / \mathrm{Y} 121 \mathrm{~F} / \mathrm{T} 289 \mathrm{~A}$. Strains 3-1-B and 3-1-C have $\mathrm{TR}_{34} / \mathrm{L} 98 \mathrm{H}$ and mutations

$155 \mathrm{~T} 289 \mathrm{~A}, \mathrm{I364V}$, and G448S. Notably, $\mathrm{TR}_{34} / \mathrm{L} 98 \mathrm{H}$ and $\mathrm{G} 448 \mathrm{~S}$ are known to play a

156 role in azole resistance, and T289A is typically accompanied by $\mathrm{TR}_{46}$ (Hagiwara

157 et al, 2016a). Thus, the Cyp51A of strains 3-1-B and 3-1-C showed complicated

158 sequence variation, including three mutations related to azole resistance.

159

Table 2. Cyp51A variation and microsatellite typing of the strains in this study

\begin{tabular}{|c|c|c|c|c|c|c|c|c|c|c|}
\hline Strain ID & Cyp51A variation & $2 A$ & 2B & $2 \mathrm{C}$ & $3 A$ & 3B & $3 \mathrm{C}$ & 4A & 4B & $4 C$ \\
\hline $3-1-A$ & $\mathrm{TR}_{46} / \mathrm{Y} 121 \mathrm{~F}, \mathrm{~T} 289 \mathrm{~A}$ & 10 & 20 & 8 & 44 & 9 & 10 & 8 & 10 & 7 \\
\hline 3-1-B & $\mathrm{TR}_{34} / \mathrm{L} 98 \mathrm{H}, \mathrm{T} 289 \mathrm{~A}, \mathrm{I364V}, \mathrm{G} 448 \mathrm{~S}$ & 23 & 10 & 9 & 35 & 9 & 6 & 8 & 10 & 18 \\
\hline $3-1-c$ & $\mathrm{TR}_{34} / \mathrm{L} 98 \mathrm{H}, \mathrm{T} 289 \mathrm{~A}, \mathrm{I364V}, \mathrm{G} 448 \mathrm{~S}$ & 23 & 10 & 9 & 35 & 9 & 6 & 8 & 10 & 18 \\
\hline 3-1-D & $\begin{array}{l}\mathrm{TR}_{46} / \mathrm{Y} 121 \mathrm{~F}, \mathrm{~T} 289 \mathrm{~A}, \mathrm{~S} 363 \mathrm{P}, 1364 \mathrm{~V}, \\
\text { G448S }\end{array}$ & 24 & 20 & 12 & 45 & 9 & 11 & 8 & 10 & 18 \\
\hline 3-1-E & $\mathrm{TR}_{46} / \mathrm{Y} 121 \mathrm{~F}, \mathrm{~T} 289 \mathrm{~A}$ & 26 & 20 & 12 & 36 & 9 & 22 & 8 & 14 & 31 \\
\hline 3-1-F & $\mathrm{TR}_{46} / \mathrm{Y} 121 \mathrm{~F}, \mathrm{~T} 289 \mathrm{~A}$ & 25 & 20 & 12 & 45 & 11 & 6 & 10 & 12 & 18 \\
\hline $3-1-G$ & $\mathrm{TR}_{46} / \mathrm{Y} 121 \mathrm{~F}, \mathrm{~T} 289 \mathrm{~A}$ & 23 & 10 & 9 & 36 & 9 & 6 & 12 & 10 & 7 \\
\hline $3-1-H$ & wt & 23 & 19 & 15 & 33 & 11 & 7 & 13 & 9 & 5 \\
\hline
\end{tabular}

160

161 Varied sensitivity to azoles in the strains from a single bulb 
162 As previously reported, strains 3-1-A to G, which have TRs in cyp51A, showed

163 VRCZ resistance $(>32 \mu \mathrm{g} / \mathrm{ml})$ in minimum inhibitory concentration tests

164 (Hagiwara, 2020). To further understand the susceptibility to azole drugs, colony

165 growth was evaluated on potato-dextrose-agar (PDA) containing $10 \mu \mathrm{g} / \mathrm{ml}$ of

166 VRCZ (Fig. 1A). Strains 3-1-B, 3-1-C, and 3-1-D were more tolerant to VRCZ

167 than the other strains. When grown on medium containing DMls (triflumizole,

168 imazalil, prochloraz, tebuconazole, epoxiconazole, or difenoconazole), strain

169 3-1-H, which harbors WT Cyp51A, showed the greatest growth inhibition among

170 the strains. Strains 3-1-B, 3-1-C, and 3-1-D were less affected by the DMIs

171 (except prochloraz) (Fig. 1B). On the basis of colony diameter measurement,

172 strains 3-1-B, 3-1-C and 3-1-D showed higher tolerance to VRCZ and DMIs than

173 strains 3-1-A, 3-1-E, 3-1-F, and 3-1-G (Fig. 1C). These results suggest that the

174 combination of TR and G448S mutation increases resistance to azole

175 compounds.

176 The expression levels of genes related to azole resistance were examined in

177 the eight strains by quantitative real-time (qRT)-PCR. Compared with strain

178 3-1- $\mathrm{H}$, which has the WT cyp51A gene, strains with a TR in the cyp51A gene

179 showed higher expression of $\operatorname{cyp51A}$ (Fig. 2A). Overexpression of $c d r 1 B$, which

180 encodes an ABC transporter, has been reported to confer azole resistance.

181 Thus, the expression level of $c d r 1 B$ was also determined in the eight strains by

182 qRT-PCR. Strains 3-1-B and 3-1-C showed relatively high expression levels of

$183 \operatorname{cdr1B}$ (Fig. 2B).

184

185 Microsatellite typing analysis of tulip bulb isolates

186 To investigate the genetic relationships between the eight strains co-isolated

187 from a single tulip bulb, microsatellite analysis using STRAf was performed

188 (Table 2). This analysis also included TR-type strains that were previously

189 reported and isolated in different countries and strains isolated from plant bulbs 
190 in Japan (Nakano et al, 2020, Hagiwara, 2020) (Fig. 3). Among the eight strains,

191 the STRAf patterns of 3-1-B and 3-1-C matched perfectly. Strain 3-1-D is closely

192 related to them, as this strain contains the same number of STRs in 4 of the 9

193 panels. Similarly, strain 3-1-D shares the same number of STRs as strain 3-1-F

194 in 4 of the 9 panels. These four strains grouped into the same clade. The other

195 strains were distantly positioned in the dendrogram. Interestingly, some strains

196 that were isolated from plant bulbs in the study by Nakano et al. (2020) showed

197 a close relationship with our strains. NGS-ER15 had an STR pattern similar to

198 that of our strains 3-1-B and 3-1-C (5 of the 9 panells), which is consistent with

199 these strains having the same Cyp51A allele $\left(\mathrm{TR}_{34} / \mathrm{L} 98 \mathrm{H} / \mathrm{T} 289 \mathrm{~A} / \mathrm{I364V} / \mathrm{G} 448 \mathrm{~S}\right)$.

200 Strains NGS-ER6 and NGS-ER7 of Nakano et al. (2020) are closely related to

201 strains 3-3-A and 3-3-B that were isolated from a single another tulip bulb in our

202 previous study (Hagiwara, 2020). Note that these extraordinarily close relatives

203 were isolated from plant bulbs in different laboratories.

204

205 Genome sequencing and comparison between strains

206 To gain more insight into genetic differences or relatedness, genomes of the

207 eight strains (3-1-A to $\mathrm{H}$ ) were sequenced using the Illumina platform. Complete

208 mitochondrial genomes were successfully obtained for the strains $(31,749$ to

20931,770 base pairs [bp] long) (Table 3). A phylogenetic tree was constructed

210 using the mitochondrial genomes and those of other strains (IFM 61407, IFM

21159365 , and IFM 61578) that had been clinically isolated in Japan

212 (Takahashi-Nakaguchi et al, 2015) (Fig. 4A). This dendrogram indicated that the

213 eight strains isolated from the tulip bulb can be divided into three groups. Group

214 m1 contains strains 3-1-A, 3-1-D, and 3-1-G; strains 3-1-B, 3-1-C, 3-1-E, and

$2153-1-\mathrm{F}$ are in Group m2. Strain 3-1-H was distantly positioned from both Group

$216 \mathrm{~m} 1$ and $\mathrm{m} 2$. Differences in the length of the mitochondrial genome well reflect

217 the grouping, suggesting that strains within each group are very close relatives. 
Table 3. Results of genome sequencing for strains isolated from a single tulip bulb

\begin{tabular}{|l|l|l|l|l|l|l|l|}
\hline Strain ID & Total length of & & G of & Mitochondrial & Mito & CSP & Mating \\
\hline 3-1-A & $28,889,155$ & proteins & genome [bp] & Group & type & type \\
\hline 3-1-B & $28,519,682$ & 49.342 & 9,492 & 31,770 & $\mathrm{~m} 1$ & t02 & mat1-1 \\
\hline 3-1-C & $28,533,261$ & 49.352 & 9,359 & 31,763 & $\mathrm{~m} 2$ & t02 & mat1-1 \\
\hline 3-1-D & $29,087,830$ & 49.355 & 9,490 & 31,763 & $\mathrm{~m} 2$ & t02 & mat1-1 \\
\hline 3-1-E & $28,703,796$ & 49.324 & 9,515 & 31,770 & $\mathrm{~m} 1$ & t02 & mat1-1 \\
\hline 3-1-F & $28,808,510$ & 49.398 & 9,464 & 31,763 & $\mathrm{~m} 2$ & t02 & mat1-1 \\
\hline
\end{tabular}

218 In the microsatellite typing analysis described above, strains 3-1-B, 3-1-C, 3-1-D,

219 and 3-1-F were grouped into the same clade, but this was inconsistent with the

220 grouping based on mitochondrial genomes, in which strain 3-1-D was not in the

221 same group as strains $3-1-B, 3-1-C$, and 3-1-F. 


\begin{tabular}{|l|l|l|l|l|l|l|l|}
\hline $3-1-\mathrm{G}$ & $29,178,518$ & 49.295 & 9,543 & 31,770 & $\mathrm{~m} 1$ & t02 & mat1-1 \\
\hline $3-1-\mathrm{H}$ & $28,716,638$ & 49.611 & 9,617 & 31,749 & $\mathrm{~m} 3$ & t01 & mat1-1 \\
\hline
\end{tabular}

222 Nuclear genomes of the eight strains were compared with the reference

223 genome of $A$. fumigatus strain Af293 (retrieved from AspGD,

224 http://www.aspgd.org/) ; 92.2\% to 93.6\% of the Af293 genome was covered in

225 the eight strains, and 69,949 to 79,391 SNPs were detected the genomes of the

226 eight strains compared with the sequence of Af293 (Table 4). Phylogenetic

227 analysis of the eight strains and previously-sequenced strains was performed by

228 using concatenated sequences of the SNP positions (Takahashi-Nakaguchi et al,

229 2015) (Fig. 4B). Among the eight strains, 3-1-H was distantly positioned in the

230 dendrogram as an independent clone. The other seven strains showed

231 moderately close genetic-relatedness to each other based on comparison with

232 the apparently independent clinical strains. Strains 3-1-B and 3-1-C showed the

233 closest relationship, which was supported by the lowest number $(6,241)$ of SNPs

234 between strains (Table 4). This is consistent with the results of microsatellite and

235 mitochondrial genome typing. Nevertheless, in the mitochondrial genome typing,

236 strain 3-1-E was in Group m2 with strains 3-1-B, 3-1-C, and 3-1-F; however,

237 strain 3-1-E was relatively distant from these three strains in phylogenetic

238 analysis based on the nuclear genome (Fig. 4C).

239 From the genome sequences, CSP typing was performed, which can typify

240 strains by sequence variation at a single locus (csp: Afu3g08990) (Klaassen et al,

241 2009). The results showed that seven strains (3-1-A to 3-1-G) carried an

242 identical type (t02), but strain 3-1-H strain had type t01. Sequence analysis for

243 mating type revealed that all but strain 3-1-F harbored mat1-1, whereas 3-1-F

244 carried mat1-2 (Table 3). 


\section{Comparison of genome-wide SNP frequency pattern}

247 Inconsistency in strain typing among the typing methods using the mitochondrial

248 and chromosomal genome sequences caused us to speculate that genetic

249 recombination had occurred between the strains isolated from the single tulip

250 bulb. To help test this hypothesis, the SNP frequency and distribution were

251 investigated and compared among the strains in a genome-wide manner (Fig.

252 S1). There were several regions where the patterns of SNP frequency markedly

253 differed among the strains (Fig.

Table 4. Summary of SNPs in strains isolated from a single tulip bulb.

\begin{tabular}{|l|l|l|l|l|l|l|l|l|l|l|}
\hline Strain ID & \% of covered & \# of SNPs & 3-1-A & 3-1-B & 3-1-C & 3-1-D & 3-1-E & 3-1-F & 3-1-G & 3-1-H \\
\hline 3-1-A & $92.7 \%$ & 74,865 & & & & & & & & \\
\hline 3-1-B & $92.2 \%$ & 77,715 & 46,380 & & & & & & & \\
\hline 3-1-C & $92.2 \%$ & 77,879 & 46,296 & 6,241 & & & & & & \\
\hline 3-1-D & $93.3 \%$ & 78,394 & 38,184 & 46,181 & 46,244 & & & & & \\
\hline 3-1-E & $92.6 \%$ & 76,218 & 50,656 & 56,072 & 55,902 & 59,081 & & & & \\
\hline 3-1-F & $93.0 \%$ & 75,553 & 40,062 & 45,535 & 45,284 & 52,533 & 54,507 & & & \\
\hline 3-1-G & $93.0 \%$ & 79,391 & 32,777 & 36,509 & 36,566 & 36,517 & 57,646 & 44,451 & & \\
\hline 3-1-H & $93.6 \%$ & 69,949 & 95,960 & 91,751 & 91,720 & 88,448 & 89,321 & 92,982 & 93,204 & \\
\hline
\end{tabular}

*1 These are relative to the genome of reference strain A. fumigatus Af293.

Table 5. The number of orthologous genes in the strains isolated from a single tulip bulb compared with reference strain A. fumigatus Af293.

\begin{tabular}{|l|l|l|}
\hline Chromosome & \# of orthologous genes compared with strain Af293 \\
\hline
\end{tabular}




\begin{tabular}{|l|l|l|l|l|l|l|l|l|l|}
\hline & Af293 & 3-1-A & 3-1-B & $3-1-\mathrm{C}$ & $3-1-\mathrm{D}$ & $3-1-\mathrm{E}$ & $3-1-\mathrm{F}$ & $3-1-\mathrm{G}$ & $3-1-\mathrm{H}$ \\
\hline chr1 & 1,642 & 1,369 & 1,355 & 1,377 & 1,371 & 1,369 & 1,365 & 1,370 & 1,370 \\
\hline chr2 & 1,640 & 1,433 & 1,406 & 1,425 & 1,425 & 1,411 & 1,436 & 1,427 & 1,433 \\
\hline chr3 & 1,395 & 1,163 & 1,163 & 1,169 & 1,179 & 1,152 & 1,173 & 1,179 & 1,189 \\
\hline $\operatorname{chr} 4$ & 1,253 & 1,089 & 1,075 & 1,093 & 1,090 & 1,098 & 1,096 & 1,085 & 1,095 \\
\hline $\operatorname{chr} 5$ & 1,367 & 1,151 & 1,156 & 1,160 & 1,153 & 1,153 & 1,162 & 1,156 & 1,153 \\
\hline $\operatorname{chr} 6$ & 1,249 & 1,068 & 1,067 & 1,074 & 1,058 & 1,069 & 1,073 & 1,070 & 1,080 \\
\hline $\operatorname{chr} 7$ & 651 & 490 & 478 & 476 & 483 & 489 & 493 & 494 & 489 \\
\hline chr8 & 628 & 502 & 496 & 507 & 497 & 505 & 506 & 491 & 513 \\
\hline Total & 9,825 & 8,265 & 8,196 & 8,281 & 8,256 & 8,246 & 8,304 & 8,272 & 8,322 \\
\hline
\end{tabular}

254 S1). For example, regions 5-A, 5-B, and 5-C on chromosome 5 were

255 particularly characteristic (Fig. 5A). In region 5-A, strains 3-1-A, 3-1-E, and 3-1-H

256 showed similar patterns of SNP frequency. In region 5-B, the pattern of strain

257 3-1-A was similar to that of strains 3-1-F and 3-1-H. In region 5-C, the pattern of

258 strain 3-1-A was similar to that of strains 3-1-E, 3-1-G, and 3-1-H. These results

259 indicate that strain 3-1-A shares parts of the sequence of chromosome 5 with

260 strains 3-1-E, 3-1-F, 3-1-G, and 3-1-H. Such intergenomic variations were also

261 found on other chromosomes (Fig. 5B, Fig. S1). These results showed a

262 genome-wide mosaic pattern of SNP frequency, which is indicative of genetic

263 recombination events in the strains.

264

265 Comparing genome-wide distribution of orthologous genes

266 To further investigate genome shuffling in the strains, we compared the

267 patterns of orthologous among the strains isolated from the tulip bulb. First, the

268 genes shared with the reference genome of $A$. fumigatus strain Af293 were

269 investigated based on reciprocal blast hits (RBHs), which resulted in the isolates

270 containing 8,196 to 8,322 orthologs of genes in strain Af293 (Table 5). The

271 positions of the orthologs were generally evenly distributed in the strains, 
272 although fewer orthologs were found on chromosome 7. Notably, different

273 patterns of ortholog content were displayed in some regions of the genomes of

274 the various strains (Fig. 6). I.e., some sets of strains have lost particular sets of

275 genes, and other sets of genes have been lost in other sets of strains. Hence,

276 the set of strains that shares an ortholog pattern is different at each locus (Fig. 6).

277 This suggests repeated genome shuffling among the strains.

278

279 Varied tolerance to agricultural fungicides

280 As these strains were derived from a horticultural product, they may have been

281 exposed to agricultural fungicides besides DMls. Hence, the susceptibility of the

282 eight strains to Qol (pyraclostrobin), SDHI (boscalid), methyl benzimidazole

283 carbamate (carbendazim), and phenylpyrrole (fludioxonil) was evaluated on

284 PDA plates. There was no significant difference among the strains in

285 susceptibility to fludioxonil and boscalid (Fig. 7A\&B). However, the colony of

286 strain 3-1-H was smaller than those of the other seven strains on the medium

287 containing pyraclostrobin or carbendazim. These results suggest that there is

288 varied tolerance to pyraclostrobin and carbendazim among the strains.

289 From the genome sequences, mutations that are possibly responsible for

290 tolerance to carbendazim and pyraclostrobin were searched in the target

291 molecules tubulin and cytochrome b that are encoded by tubA (Afu1g10910) and

292 cytB (AfuMt00001), respectively (Fig. 6C). The amino acid substitution F219Y

293 was found in TubA of strains 3-1-A to 3-1-G. This substitution has been reported

294 in several carbendazim-resistant strains of plant pathogenic fungi (Yarden and

295 Katan, 1993, Zhou et al, 2020). To investigate how the mutation is distributed in

296 human pathogenic $A$. fumigatus genomes, the SNP database in FungiDB was

297 explored. According to the dataset, 18\% (14 of 77 strains) of $A$. fumigatus

298 contain F219Y in TubA. Notably, eight of the 77 strains were isolated from the

299 environment, and four of these possess the amino acid substitution. 
300 In CytB, mutations V13I and G143A were found in strains 3-1-A to 3-1-G, 301 and V119I was found in 3-1-H. G143A in CytB has been reported to confer the

302 resistance to Qol in many plant pathogenic fungi (Samuel et al, 2011, Bolton et

303 al, 2012), suggesting that this mutation in A. fumigatus is related to low

304 sensitivity to Qol fungicide. As mitochondrial genome sequences are scarce in

305 public databases, we investigated the sequence of the cytb gene in nine strains

306 that were clinically isolated in a previous study and whose mitochondrial genome

307 sequence is at least partly available (Takahashi-Nakaguchi et al, 2015). Among

308 these nine strains, no G143A mutation was observed, whereas seven of the

309 strains contain V119l (as also observed in our strain 3-1-H).

310

311 Discussion

312 The distribution of azole-resistant $A$. fumigatus in natural environments has

313 drawn increasing attention in recent years, with special interest in where the

314 resistant strains have emerged, inhabit, and have been translocated to. However,

315 deep understanding is still lacking. In this work, to fill in gaps in knowledge, we

316 focused on strains that were isolated from a single tulip bulb.

317 Sexual reproduction of $A$. fumigatus was demonstrated in laboratory

318 conditions in 2009 (O'Gorman et al., 2009). After this discovery, researchers

319 paid more attention to the pan-genome of clinical isolates of this pathogenic

320 fungus. However, gene flow in the environment has been poorly studied.

321 Population genetics study using linkage disequilibrium analysis for genetic

322 markers supported the view that $A$. fumigatus reproduces asexually and sexually

323 in natural habitats (Klaassen et al., 2012). However, although there is

324 accumulating evidence for genetic recombination in nature, proving the

325 occurrence of sexual reproduction is difficult unless one can directly collect

326 cleistothecia and ascospores formed in the environment. Genetic recombination

327 in sexual development is suggested to cause the emergence of TR mutation in 
328 cyp51A gene through unequal crossover (Zhang et al., 2017). Thus, sexual

329 reproduction is considered both to spread mutations by fusion with other strains

330 and production of progeny, and to locally produce de novo TR mutations, which

331 could affect the prevalence of resistance to drugs and fungicides. Our data show

332 that seven of the eight isolates from a single tulip bulb contain TR mutations, and

333 the genetic variation between the TR-containing strains is low compared with

334 that between apparently independent strains. In addition, on the basis of

335 genome-wide distributions of SNPs and orthologous genes, genetic

336 recombination is likely to have occurred between the seven strains.

337 Co-isolation of the strains from a single bulb indicates that they have had

338 much opportunity to physically interact with each other inside or on the bulb. In

339 addition to the close spatial relationship of the fungal strains, they may interact

340 for a long time. In the conventional process of plant bulb production, bulbs are

341 multiplied from a parental bulb. This bulb multiplication is continued every year,

342 which presumably causes the sustained presence of the fungi on/inside the

343 bulbs. As described here, several strains were attached to a single bulb. These

344 strains might have encountered others and genetically mixed many times. Once

345 mutations giving rise to resistance to azoles emerged, the mutations could be

346 preferentially and stably retained in the microbial community inside the bulb.

347 Sequencing analysis of the eight strains produced complete mitochondrial

348 genomes and chromosomal genomes. The mitochondrial genome was of great

349 help in interpreting whether there had been sexual reproduction among the

350 strains. On the basis of mitochondrial genome sequences, the strains with TR

351 mutation can be classed into Groups m1 and m2 (Fig. 4A). The length and

352 sequences of the mitochondrial genomes are highly conserved in each group,

353 indicating that they are genetically close progenies. However, the chromosomal

354 genomes were diverse among the strains with TR mutation to the extent that

355 there were 32,000 to 59,000 SNPs, excepting strains 3-1-B and 3-1-C which had 
356 approximately 6,200 SNPs (Table 4). The differences in grouping based on

357 mitochondrial and chromosomal genomes strongly suggested a genetic

358 recombination event. We therefore propose that the complete mitochondrial

359 genome is valuable for gaining deeper insight into genetic relatedness among

360 and between environmental and clinical isolates.

361 Strains with complicated cyp51A alleles have been reported in the literature

362 and this paper (Table 1). For instance, we have isolated $A$. fumigatus strains

363 with $\mathrm{TR}_{34} / \mathrm{L} 98 \mathrm{H} / \mathrm{T} 289 \mathrm{~A} / \mathrm{I364V} / \mathrm{G} 448 \mathrm{~S}(3-1-\mathrm{B}$ and 3-1-C) and

$364 \mathrm{TR}_{46} / \mathrm{Y} 121 \mathrm{~F} / \mathrm{T} 289 \mathrm{~A} / \mathrm{S} 363 \mathrm{P} / \mathrm{I364V/G448S} \mathrm{(3-1-D)} \mathrm{mutations} \mathrm{in} \mathrm{the} \mathrm{cyp51A} \mathrm{gene.}$

$365 \mathrm{TR}_{34} / \mathrm{L} 98 \mathrm{H}$ is a typical TR-type mutation conferring resistance to ITCZ and in

366 some cases to $\mathrm{VRCZ}$, whereas $\mathrm{TR}_{46} / \mathrm{Y} 121 \mathrm{~F} / \mathrm{T} 289 \mathrm{~A}$ confers resistance to VRCZ

367 and in most cases to ITCZ (van Ingen et al, 2015, Buil et al, 2018). Amino acid

368 substitution G448S contributes to resistance to VRCZ and occasionally to ITCZ

369 (Bellete et al, 2010, Toyotome et al, 2016, Cao et al, 2020). Our finding that

370 three strains (3-1-B, 3-1-C, and 3-1-D) showed a higher tolerance to VRCZ and

371 some DMls than strains with only $\mathrm{TR}_{46} / \mathrm{Y} 121 \mathrm{~F} / \mathrm{T} 289 \mathrm{~A}$ mutation is suggestive of

372 elevation of tolerance to azole drugs by combining mutations. Importantly,

373 strains with G448S mutation have been isolated not only from clinical samples

374 but also from soil (Cao et al, 2020). We cannot rule out the possibility that the

375 G448S mutation originally emerged and was retained in strains with

$376 \mathrm{TR}_{46} / \mathrm{Y} 121 \mathrm{~F} / \mathrm{T} 289 \mathrm{~A}$ under the selective pressure of fungicides.

377 The $A$. fumigatus strains used in the present work were isolated from a tulip

378 bulb by culturing at $45^{\circ} \mathrm{C}$ on plates containing medium supplemented with

379 fluconazole to select fungi that were resistant to fluconazole (Hagiwara 2020). In

380 total in that study, $A$. fumigatus was isolated from $50.8 \%$ of tulip bulbs $(96 / 189)$,

381 and strains isolated from $20.6 \%$ of the bulbs (39/189) had TR mutation. Because

382 A. fumigatus is a saprophytic fungus that widely inhabits soil, compost, plant

383 debris, wood chips, the air, and aquatic environments, it was not surprising that 
384 half of the tulip bulbs were contaminated with $A$. fumigatus. However, we have

385 no idea how the fungus resides on or inside the bulbs from a biological viewpoint.

386 Because of the high frequency of $A$. fumigatus isolation from tulip bulbs, there

387 might be certain mechanisms by which $A$. fumigatus colonizes and infects the

388 plant tissue, enabling persistence across bulb progenies. Notably, some $A$.

389 fumigatus strains were isolated from Citrus macrocarpa, Myricaria laxiflora,

390 Ligusticum wallichii, and Moringa oleifera (Francisco et al, 2020, Qin et al, 2019,

391 Li et al, 2020, Arora and Kaur, 2019) as an endophyte. In general, however, the

392 view that $A$. fumigatus has an endophytic mode in its life cycle remains to be

393 established. In consideration of the dynamic mobilization of $A$. fumigatus in the

394 environment, its association with plants may be overlooked, and we should pay

395 more attention to it.

396 Recently, several field studies were published in which the prevalence of

397 azole-resistant $A$. fumigatus was investigated in association with fungicide use.

398 Work by Zhou et al. (2021) demonstrated that the concentration of triazoles in

399 the soil of greenhouses was not significantly correlated with azole susceptibility

400 of isolates. In another study from Germany, a low frequency of azole-resistant

401 isolates from crop fields was reported regardless of azole fungicide use (Barber

402 et al, 2020). A study by Frasije et al. (2020) also reported a low number of

403 azole-resistant isolates in the soils of wheat-cropping fields subjected to

404 fungicide treatment. The authors considered that arable crop production is low

405 risk for development of azole resistance. Conversely, a large-scale survey

406 across China was conducted, which showed that the residual level of azole

407 fungicides in paddy soils positively correlated with the prevalence of

408 azole-resistant $A$. fumigatus (Cao et al, 2021). Field research on azole-resistant

409 A. fumigatus has started in many countries. More studies are required on the

410 effects of fungicide use on the occurrence and spread of azole-resistant $A$.

411 fumigatus in the environment, including agricultural and horticultural settings. 
412 In plant bulbs, there may be other pathogenic and nonpathogenic fungi

413 beside $A$. fumigatus. They are also exposed to fungicides when the bulbs are

414 treated with fungicide. Repeated use of fungicides would facilitate the

415 occurrence of resistance mutations in non-targeted fungi as well as in the target

416 fungi of the pesticide. In the present study, we found that mutations in CytB and

417 TubA that are related to resistance to Qol and carbendazim fungicides,

418 respectively, were detected in $A$. fumigatus strains as an example of non-target

419 fungi. These mutations might have been resulted from fungicide exposure during

420 bulb production. Importantly, identical mutations of $A$. fumigatus were reported

421 by Fraaije et al. (2020) and are found in database. These findings suggest that

422 mutations related to resistance to antifungal agents are already present in the

423 genomes of environmental fungi regardless of their pathogenicity. The boundary

424 between acquired and natural resistance to antifungal compounds may become

425 unclear in the near future.

426

427 Materials and Methods

428 Strains and culture conditions

429 Strains 3-1-A to $3-1-\mathrm{H}$ used in this study were obtained in previous study and

430 were isolated from a single tulip bulb (Hagiwara, 2020). For plate and liquid

431 cultures, PDA and potato-dextrose broth (PDB) were used, respectively. For

432 colony growth tests, $10^{5}$ conidia of each strain were inoculated and incubated for

$43348 \mathrm{~h}$ at $37^{\circ} \mathrm{C}$ before taking pictures. Insusceptibility tests, $10 \mu \mathrm{g} / \mathrm{ml} \mathrm{VRCZ,}$

434 imazalil, prochloraz, triflumizole, tebuconazole, epoxiconazole, and

435 difenoconazole were respectively added to PDA. The control plate contained the

436 equivalent volume of dimethylsulfoxide (DMSO). For measuring colony diameter,

437 the culture time was 28 or $30 \mathrm{~h}$. The data were obtained in triplicate, and the

438 mean and standard deviation are presented. The fungicides fludioxonil, 
439 carbendazim, boscalid, and pyraclostrobin were used at $0.2 \mu \mathrm{g} / \mathrm{ml}, 5 \mu \mathrm{g} / \mathrm{ml}, 2.5$

$440 \mu \mathrm{g} / \mathrm{ml}$, and $10 \mu \mathrm{g} / \mathrm{ml}$, respectively.

441

\section{Quantitative real-time RT-PCR}

443 Strains were cultured in $\mathrm{PDB}$ at $37^{\circ} \mathrm{C}$ for $18 \mathrm{~h}$ and harvested. The mycelia were

444 frozen in liquid nitrogen, and total RNA was isolated using Sepasol Super G

445 (Nacalai Tesque, Kyoto, Japan). cDNA was obtained by reverse transcription

446 reaction using the total RNA sample and ReverTra Ace qPCR RT Master Mix

447 with gDNA remover (TOYOBO, Osaka, Japan).

448 Real-time RT-PCR was performed using Brilliant III Ultra-Fast SYBR Green

449 QPCR Master Mix (Agilent Technologies, Inc., Santa Clara, CA, USA) as

450 described previously (Ninomiya et al, 2020). Relative expression ratios were

451 calculated using the comparative cycle threshold (Ct) method. The

452 actin-encoding gene was used as a normalization reference. Each sample was

453 tested in triplicate, and the standard deviation is presented. The primer sets

454 used were described in Hagiwara et al, 2017.

455

456 Microsatellite typing

457 Microsatellite typing was performed as described previously (Hagiwara et al,

458 2014). Briefly, nine microsatellite regions of approximately 400 bp were PCR

459 amplified using purified genome DNA as a template and sequenced by the

460 Sanger method. The repeat numbers of each locus were counted from the

461 sequences. A dendrogram was constructed using Cluster 3.0 by hierarchical

462 clustering with City-block distance for average linkage, and drawn using

463 Treeview ver. 1.1.6r2 (de Hoon et al, 2004, Saldanha, 2004).

465 Genome sequencing 
466 Whole-genome sequencing using next-generation methods was performed as

467 described previously (Hagiwara et al., 2018). In brief, we extracted genomic

468 DNA from overnight-cultured mycelia with NucleoSpin Plant II (Takara Bio,

469 Shiga, Japan). For paired-end library preparation, an NEBNext Ultra DNA

470 Library Prep Kit (New England BioLabs, MA, USA) and NEBNext Multiplex

471 Oligos (New England BioLabs) were used in accordance with the manufacturer's

472 instructions. A total of 11 strains including 3-1-A to 3-1-H, IFM 59365, IFM 61407,

473 and IFM 61578 were sequenced. Paired-end sequencing (150-bp) on a HiSeq

4744000 system (Illumina, San Diego, CA, USA) was carried out by GENEWIZ

475 (South Plainfield, NJ, USA).

476

477 SNP detection

478 In addition to the abovementioned 11 strains, we used raw data for seven strains

479 for comparison, which have been taken in a study by Takahashi-Nakaguchi et al

480 (2015). Adapters and low-quality bases from Illumina reads were trimmed by

481 fastp (ver. 0.20.1) (Chen et al., 2018). Filtered reads were aligned against the $A$.

482 fumigatus strain Af293 reference genome using BWA (ver. 0.7.17-r1188) (Li and

483 Durbin 2009). SNP detection was performed as described previously (Hagiwara

484 et al., 2018). Briefly, SNPs were identified by using SAMtools (ver. 1.9) (Li et al.,

4852009 ) and filtered with >20-fold coverage, $>30$ mapping quality, and $75 \%$

486 consensus using in-house scripts (Suzuki et al., 2014; Tenaillon et al., 2012).

487

488 Phylogenetic tree construction

489 Among the strains that were sequenced, mitochondrial genomes of 12 strains

490 were available and aligned by MAFFT (ver. 7.475) (Katoh and Standley, 2013).

491 A phylogenetic tree was constructed using multithreaded RAxML (ver. 8.2.12)

492 (Stamatakis, 2014), the GTRCAT model, and 1,000 bootstrap replicates, and

493 visualized by iTOL (Letunic and Bork 2019). For chromosomal genome 
494 phylogenetic classification, 90,987 polymorphic loci were predicted from 18

495 strains and concatenated, then used for construction of a phylogenetic tree by

496 the methods described above.

497

\section{Genome assembly and gene prediction}

499 Mitochondrial genomes were assembled and annotated using GetOrganelle (ver.

500 1.6.4) (Jin et al., 2020) and MITOS2 (Bernt et al., 2013), respectively. To filter

501 the mitochondrial reads, trimmed reads were aligned against mitochondrial

502 genomes by BWA (ver. 0.7.17-r1188) (Li and Durbin, 2009), and the mapped

503 reads were filtered by SAMtools (ver. 1.9) (Li et al., 2009) and SeqKit (Shen et

504 al., 2016). Contigs were assembled by VelvetOptimiser (ver. 2.2.6) (Zerbino and

505 Birney 2008), followed by generation of a simulated mate-paired library using

506 wgsim (ver. 0.3.1-r13) (https://github.com/lh3/wgsim). The assembly of nuclear

507 genomes was carried out by ALLPATHS-LG (ver. R52488) (Gnerre et al., 2011).

508 The annotation of assembled nuclear genomes was performed by the

509 Funannotate pipeline (ver. 1.7.4) (https://funannotate.readthedocs.io/en/latest/)

510 as described previously (Takahashi et al., 2021). Following identification of

511 repeat sequences by RepeatModeler (ver. 1.0.11)

512 (http://www.repeatmasker.org/RepeatModeler.html) and RepeatMasker (ver.

513 4.0.7) (https://www.repeatmasker.org), Funannotate ab initio prediction was

514 performed with the option “--busco_seed_species=aspergillus_fumigatus” by

515 Augustus (ver. 3.3.3) (Stanke et al., 2006), GeneMark-ES (ver. 4.38)

516 (Ter-Hovhannisyan et al., 2008), GlimmerHMM (ver. 3.0.4) (Majoros et al., 2004),

517 and SNAP (ver. 2006-07-28) (lan, 2004) using exon hints from the proteins of $A$.

518 fumigatus Af293 and N. fischeri NRRL 181 downloaded from the Aspergillus

519 Genome Database (http://www.aspgd.org/) (Cerqueira et al., 2014). The

520 completeness of draft genomes and predicted proteins was evaluated by 
521 BUSCO (ver. 4.0.6) (Seppey et al., 2019) with the database eurotiales_odb10.

522 Most tools were obtained through Bioconda (Grüning et al., 2018).

523

524 Detection of orthologous genes

525 Orthologous relationships with $A$. fumigatus Af293 were determined by $\mathrm{RBH}$ with

526 criteria BLASTp (ver. 2.9.0+) coverage $>80 \%$ and identity $>80 \%$ (Camacho et al., 527 2009).

528

529 Visualization of genome-wide distribution of SNPs and orthologous genes

530 SNP frequency in each 1-kb window was calculated and plotted in 250-bp steps

531 using Python (Van Rossum and Drake 2009) and R (R Core Team 2019) scripts.

532 The orthologous genes of $A$. fumigatus Af293 in each strain were visualized by $R$ 533 script.

534

\section{Data availability}

536 The genome sequencing data are deposited to DDBJ as DRA011961.

537 BioSample accession(s): SAMD00322244-SAMD00322251.

539 Author contributions: HT and DH designed the research; HT, SO, YK, SU, and

540 DH performed experiments; HT contributed new materials/tools; $H$ T and DH

541 analyzed data; and HT and DH wrote the manuscript.

542

543 ACKNOWLEDGMENTS

544 This study was supported by a grant from the Institute for Fermentation, Osaka

545 (to DH). HT was partly supported by the National Bioscience Database Center

546 (NBDC) of the Japan Science and Technology Agency (JST), and JSPS

547 KAKENHI Grant Numbers 21K07001 and 16H06279. DH and HT were partly 
548 supported by AMED, Grant Number JP19fm0208024. We thank Edanz

549 (https://jp.edanz.com/ac) for editing a draft of this manuscript. The authors

550 declare no competing interest.

551

\section{$552 \quad$ Figure legends}

553 Fig. 1. Colony growth of Aspergillus fumigatus strains isolated from a single tulip

554 bulb on potato-dextrose-agar (PDA) containing azoles. (A) Growth on PDA

555 containing voriconazole (VRCZ). Each strain was inoculated on PDA with

556 dimethylsulfoxide (DMSO) as a control or $10 \mu \mathrm{g} / \mathrm{ml} \mathrm{VRCZ,} \mathrm{and} \mathrm{was} \mathrm{incubated} \mathrm{for}$

55748 h. (B) Growth on PDA containing demethylase inhibitors (DMIs). Each strain

558 was inoculated on PDA with DMSO as a control or $10 \mu \mathrm{g} / \mathrm{ml} \mathrm{DMI}$, and was

559 incubated for 48 h. (C) Colony diameter on PDA containing VRCZ or DMls. Each

560 strain was inoculated on PDA with DMSO as a control or $10 \mu \mathrm{g} / \mathrm{ml}$ azole, and

561 incubated for 28 h. Error bars represent standard deviations based on three

562 independent replicates.

563

564 Fig. 2. Gene expression analysis by quantitative real-time (qRT)-PCR.

565 Expression levels of $\operatorname{cyp51A}(\mathrm{A})$ and $\operatorname{cdr} 1 B(\mathrm{~B})$ were determined in the eight

566 strains isolated from a single tulip bulb. The strains were cultured in

567 potato-dextrose broth for $18 \mathrm{~h}$. The actin gene was used as an internal control.

568 Error bars represent standard deviations based on three independent replicates.

569

570 Fig. 3. Microsatellite-typing analysis of $A$. fumigatus strains with tandem repeat

571 (TR) mutations. The dendrogram was constructed using short tandem repeat for

572 A. fumigatus (STRAf) patterns of the strains. The nine STR panels are shown.

573 The strains listed refer to the literature (Chen et al, 2019, Hagiwara et al, 2016b,

574 Nakano et al, 2020, Hagiwara, 2020). The names of strains isolated from plant

575 bulbs in this study or other study are highlighted in pale blue or yellow, 
576 respectively. Cyp51A alleles with $\mathrm{TR}_{34}$ are indicated in red, and those with $\mathrm{TR}_{46}$ 577 in blue.

578

579 Fig. 4. Phylogenetic trees constructed using mitochondrial (A) and nuclear (B)

580 genomes. The trees were constructed using genomes of strains isolated from a

581 single tulip bulb (3-1-A to 3-1-H) or clinically isolated in a previous study

582 (Takahashi-Nakaguchi et al, 2016), as well as $A$. fumigatus reference strain

583 Af293.

584

585 Fig. 5. Differences in patterns of single nucleotide polymorphism (SNP)

586 frequency among the strains isolated from a single tulip bulb. (A) The SNP

587 presence patterns are compared in certain regions on chromosome 5 (5-A, 5-B,

588 and $5-C)$. (B) The patterns in each region could typically be divided into two

589 groups, which are indicated by yellow or blue panels. Ten genomic loci are

590 shown and compared among the eight strains.

591

592 Fig. 6. Visualization of genome-wide orthologous gene content in the strains

593 isolated from a single tulip bulb. Orthologous genes were searched against the

594 reference genome of $A$. fumigatus strain Af293. The presence of an ortholog is

595 indicated by colored ribbons for each strain (3-1-A to 3-1-H). Some regions are

596 enlarged to enable easier comparison of the patterns of gene content.

597

598 Fig. 7. Colony growth of $A$. fumigatus isolated from a single tulip bulb on PDA

599 containing fungicide. (A) Growth on PDA containing fungicide. Each strain was

600 inoculated onto PDA with DMSO as a control, or Qol (pyraclostrobin; $10 \mu \mathrm{g} / \mathrm{ml}$ ),

601 SDHI (boscalid; $2.5 \mu \mathrm{g} / \mathrm{ml}$ ), methyl benzimidazole carbamate (carbendazim; 5

$602 \mu \mathrm{g} / \mathrm{ml}$ ), or phenylpyrrole (fludioxonil; $0.2 \mu \mathrm{g} / \mathrm{ml}$ ), and incubated for $48 \mathrm{~h}$. (B)

603 Colony diameter on PDA containing fungicide. Each strain was incubated for 30 
604 h. Error bars represent standard deviations based on three independent

605 replicates. (C) Amino acid substitutions detected in CytB and TubA of $A$.

606 fumigatus strains isolated from a tulip bulb.

607

\section{Supporting Information}

609 Figure S1. Genome-wide SNP frequency compared among the eight strains

610 (3-1-A to 3-1-H). The 10 regions where the pattern is characteristically distinct

611 among the strains are marked by red boxes.

612

613 References

614 Ahangarkani, F., Badali, H., Abbasi, K., Nabili, M., Khodavaisy, S., de Groot, T., and Meis, J.F.

615 (2020) Clonal Expansion of Environmental Triazole Resistant Aspergillus fumigatus in Iran. $J$

616 Fungi (Basel) 6: 199.

617

618 Ahangarkani, F., Puts, Y., Nabili, M., Khodavaisy, S., Moazeni, M., Salehi, Z, et al. (2020) First

619 azole-resistant Aspergillus fumigatus isolates with the environmental TR ${ }_{46} / \mathrm{Y} 121 \mathrm{~F} / \mathrm{T} 289 \mathrm{~A}$

620 mutation in Iran. Mycoses 63: 430-436.

621

622 Saldanha, A.J. (2004) Java Treeview—extensible visualization of microarray data,

623 Bioinformatics 20: 3246-3248.

624

625 Alvarez-Moreno, C., Lavergne, R.A., Hagen, F., Morio, F., Meis, J.F., and Le Pape, P. (2017)

626 Azole-resistant Aspergillus fumigatus harboring $\mathrm{TR}_{34} / \mathrm{L} 98 \mathrm{H}, \mathrm{TR}_{46} / \mathrm{Y} 121 \mathrm{~F} / \mathrm{T} 289 \mathrm{~A}$ and $\mathrm{TR}_{53}$

627 mutations related to flower fields in Colombia. Sci Rep 7: 45631.

628

629 Arora, D.S., and Kaur, N. (2019) Antimicrobial Potential of Fungal Endophytes from Moringa

630 oleifera. Appl Biochem Biotechnol 187: 628-648.

631

632

633

634

635

636

637

638

639 Bernt, M., Donath, A,, Jühling, F., Externbrink, F., Florentz, C., Fritzsch, G., et al. (2012) MITOS:

640 improved de novo metazoan mitochondrial genome annotation. Mol Phylogenet Evol 69:

641 313-319.

642 
643 Bolton, M.D., Rivera, V., and Secor, G. (2013) Identification of the G143A mutation associated

644 with Qol resistance in Cercospora beticola field isolates from Michigan, United States. Pest

645 Manag Sci 69: 35-39.

646

647 Buil, J.B., Hagen, F., Chowdhary, A., Verweij, P.E., and Meis, J.F. (2018) Itraconazole,

648 Voriconazole, and Posaconazole CLSI MIC Distributions for Wild-Type and Azole-Resistant

649 Aspergillus fumigatus Isolates. J Fungi (Basel) 4: 103.

650

651 Camacho, C., Coulouris, G., Avagyan, V., Ma, N., Papadopoulos, J., Bealer, K., and Madden, T.

652 L. (2009) BLAST+: architecture and applications. BMC Bioinformatics 10: 421. doi:

653 10.1186/1471-2105-10-421

654

655

Camps, S.M., Dutilh, B.E., Arendrup, M.C., Rijs, A.J., Snelders, E., Huynen, M.A., et al. (2012)

656 Discovery of a HapE mutation that causes azole resistance in Aspergillus fumigatus through

657 whole genome sequencing and sexual crossing. PLoS One 7: e50034.

658

659

Cao, D., Wang, F., Yu, S., Dong, S., Wu, R., Cui, N., et al. (2021) Prevalence of Azole-Resistant

660 Aspergillus fumigatus is Highly Associated with Azole Fungicide Residues in the Fields. Environ

661 Sci Technol 55: 3041-3049.

662

663 Cao, D., Wu, R., Dong, S., Wang, F., Ju, C., Yu, S., et al. (2020) Five-Year Survey (2014 to

664 2018) of Azole Resistance in Environmental Aspergillus fumigatus Isolates from China.

665 Antimicrob Agents Chemother 64: e00904-20.

666

667

Cerqueira, G.C., Arnaud, M.B., Inglis, D.O., Skrzypek, M.S., Binkley, G., Simison, M., et al. (2014).

668 The Aspergillus Genome Database: multispecies curation and incorporation of RNA-Seq data to

669 improve structural gene annotations. Nucl Acids Res 42: D705-D710.

670

671 Chen, Y.C., Kuo, S.F., Wang, H.C., Wu, C.J., Lin, Y.S., Li, W.S., and Lee, C.H. (2019) Azole

672 resistance in Aspergillus species in Southern Taiwan: An epidemiological surveillance study.

673 Mycoses 62: 1174-1181.

674

675 Chen, S., Zhou, Y., Chen, Y., and Gu, J. (2018) fastp: an ultra-fast all-in-one FASTQ

676 preprocessor. Bioinformatics 34: i884-i890.

677

678 Chowdhary, A., Kathuria, S., Xu, J., Sharma, C., Sundar, G., Singh, P.K., et al. (2012) Clonal

679 expansion and emergence of environmental multiple-triazole-resistant Aspergillus fumigatus

680 strains carrying the $\mathrm{TR}_{34} / \mathrm{L} 98 \mathrm{H}$ mutations in the cyp51A gene in India. PLoS One 7: e52871.

681

682 Dunne, K., Hagen, F., Pomeroy, N., Meis, J.F., and Rogers, T.R. (2017) Intercountry Transfer of

683 Triazole-Resistant Aspergillus fumigatus on Plant Bulbs. Clin Infect Dis 65: 147-149.

684

685 Fisher, M.C., Hawkins, N.J., Sanglard, D., and Gurr, S.J. (2018) Worldwide emergence of

686 resistance to antifungal drugs challenges human health and food security. Science 360 :

$687 \quad 739-742$.

688 
689 Fraaije, B., Atkins, S., Hanley, S., Macdonald, A., and Lucas, J. (2020) The Multi-Fungicide

690 Resistance Status of Aspergillus fumigatus Populations in Arable Soils and the Wider European

691 Environment. Front Microbiol 11: 599233.

692

693 Fraczek, M.G., Bromley, M., Buied, A., Moore, C.B., Rajendran, R., Rautemaa, R., et al. (2013)

694 The $c d r 1 B$ efflux transporter is associated with non-cyp51a-mediated itraconazole resistance in

695 Aspergillus fumigatus. J Antimicrob Chemother 68: 1486-1496.

696

697 Francisco, J.C.E., Rivera, W.L., and Vital, P.G. (2020) Influences of carbohydrate, nitrogen, and

698 phosphorus sources on the citric acid production by fungal endophyte Aspergillus fumigatus

699 P3I6. Prep Biochem Biotechnol 50: 292-301.

700

701

Gnerre, S., Maccallum, I., Przybylski, D., Ribeiro, F.J., Burton, J.N., Walker, B.J., et al. (2011).

702 High-quality draft assemblies of mammalian genomes from massively parallel sequence data.

703 Proc Natl Acad Sci U. S. A. 108: 1513-1518.

704

705

Grüning, B., Dale, R., Sjödin, A., Chapman, B.A., Rowe, J., Tomkins-Tinch, C. H., et al. (2018).

706 Bioconda: sustainable and comprehensive software distribution for the life sciences. Nat Methods

707 15: 475-476.

708

709 Hagiwara, D., Miura, D., Shimizu, K., Paul, S., Ohba, A., Gonoi, T., et al. (2017) A Novel

710 Zn2-Cys6 Transcription Factor AtrR Plays a Key Role in an Azole Resistance Mechanism of

711 Aspergillus fumigatus by Co-regulating cyp51A and cdr1B Expressions. PLoS Pathog 13:

712 e1006096.

713

714

715

Hagiwara, D., Takahashi, H., Fujimoto, M., Sugahara, M., Misawa, Y., Gonoi, T., et al. (2016b)

716

Multi-azole resistant Aspergillus fumigatus harboring Cyp51A TR ${ }_{46} / \mathrm{Y} 121 \mathrm{~F} / \mathrm{T} 289 \mathrm{~A}$ isolated in

717

718 Hagiwara, D., Takahashi, H., Watanabe, A., Takahashi-Nakaguchi, A., Kawamoto, S., Kamei, K.,

719 and Gonoi, T. (2014) Whole-genome comparison of Aspergillus fumigatus strains serially

720 isolated from patients with aspergillosis. J Clin Microbiol 52: 4202-4209.

721

722

723

724

725

726

727

728

729

730

731

732

733

734

Hagiwara, D., Watanabe, A., Kamei, K., and Goldman, G.H. (2016a) Epidemiological and genomic landscape of azole resistance mechanisms in Aspergillus fungi. Front Microbio/ 21: 1382.

Hagiwara, D. (2020) Isolation of azole-resistant Aspergillus fumigatus from imported plant bulbs in Japan and the effect of fungicide treatment. J Pestic Sci 45: 147-150.

Hagiwara, D., Arai, T., Takahashi, H., Kusuya, Y., Watanabe, A., and Kamei, K. (2018) Non-cyp51A Azole-Resistant Aspergillus fumigatus Isolates with Mutation in HMG-CoA Reductase. Emerg Infect Dis 24: 1889-1897.

Hortschansky, P., Misslinger, M., Mörl, J., Gsaller, F., Bromley, M.J., Brakhage, A.A., et al. (2020) Structural basis of $\mathrm{HapE}^{\mathrm{P} 8 \mathrm{LL}}$-linked antifungal triazole resistance in Aspergillus fumigatus. 735 Life Sci Alliance 3: e202000729. 
737 Howard, S.J., Cerar, D., Anderson, M.J., Albarrag, A., Fisher, M.C., Pasqualotto, A.C., et al.

738 (2009) Frequency and evolution of Azole resistance in Aspergillus fumigatus associated with

739 treatment failure. Emerg Infect Dis. 15: 1068-1076.

740

741

742

743

lan, K. (2004). Gene finding in novel genomes. BMC Bioinformatics 5: 59.

744

Jeanvoine, A., Rocchi, S., Bellanger, A.P., Reboux, G., and Millon, L. (2020) Azole-resistant

745

Aspergillus fumigatus: A global phenomenon originating in the environment? Med Mal Infect 50:

746

747 389-395.

748

Jenks, J.D., and Hoenigl, M. (2018) Treatment of Aspergillosis. J Fungi (Basel) 4: 98.

749

Jin, J.J., Yu, W.B., Yang, J.B., Song, Y., de Pamphilis, C.W., Yi, T.S., et al. (2020).

750

751

752

753

GetOrganelle: a fast and versatile toolkit for accurate de novo assembly of organelle genomes.

Genome Biol 21: 241.

754

Katoh, K., and Standley, D. M. (2013). MAFFT multiple sequence alignment software version 7:

755

improvements in performance and usability. Mol Biol Evol 30: 772-780.

756 Klaassen, C.H.W., de Valk, H.A., Balajee, S.A., and Meis, J.F.G.M. (2009) Utility of CSP typing

757 to sub-type clinical Aspergillus fumigatus isolates and proposal for a new CSP type

758 nomenclature. J Microbiol Methods 77: 292-296.

759

760

Klaassen, C.H.W., Gibbons, J.G., Fedorova, N.D., Meis, J.F., and Rokas, A. (2012) Evidence for

761

762

763

764 genetic differentiation and variable recombination rates among Dutch populations of the opportunistic human pathogen Aspergillus fumigatus. Mol Ecol 21: 57-70.

765

Lestrade, P.P.A., Meis, J.F., Melchers, W.J.G., and Verweij, P.E. (2019) Triazole resistance in

766

Aspergillus fumigatus: recent insights and challenges for patient management. Clin Microbiol

767

768 Infect 25: 799-806.

769

770

771

772

773

774

Letunic, I., and Bork, P. (2019). Interactive Tree Of Life (iTOL) v4: recent updates and new developments. Nucl. Acids Res. 47, W256-W259.

Li, S., Chen, J.F., Qin, L.L., Li, X.H., Cao, Z.X., Gu, Y.C., et al. (2020) Two new sesquiterpenes produced by the endophytic fungus Aspergillus fumigatus from Ligusticum wallichii. $J$ Asian Nat Prod Res 22: 138-143.

775 Li, H., Handsaker, B., Wysoker, A., Fennell, T., Ruan, J., Homer, N., et al. (2009). The Sequence

776 Alignment/Map format and SAMtools. Bioinformatics 25: 2078-2079.

777

778 Li, H., and Durbin, R. (2009) Fast and accurate short read alignment with Burrows-Wheeler 779 transform. Bioinformatics 25: 1754-1760.

780

Majoros, W.H., Pertea, M., and Salzberg, S.L. (2004). TigrScan and GlimmerHMM: two open 782 source ab initio eukaryotic gene-finders. Bioinformatics 20: 2878-2879.

783 
784 McKenna, A., Hanna, M., Banks, E., Sivachenko, A., Cibulskis, K., Kernytsky, A., et al. (2010)

785 The Genome Analysis Toolkit: a MapReduce framework for analyzing next-generation DNA

786 sequencing data. Genome Res 20: 1297-1303.

787

788 Nakano, Y., Tashiro, M., Urano, R., Kikuchi, M., Ito, N., Moriya, E., et al. (2020) Characteristics of

789 azole-resistant Aspergillus fumigatus attached to agricultural products imported to Japan. $J$

790 Infect Chemother 26: 1021-1025.

791

792

Ninomiya, A., Urayama, S.I., Suo, R., Itoi, S., Fuji, S.I., Moriyama, H., and Hagiwara, D. (2020)

793 Mycovirus-Induced Tenuazonic Acid Production in a Rice Blast Fungus Magnaporthe oryzae.

794 Front Microbiol 11: 1641.

795

796

797

Nywening, A.V., Rybak, J.M., Rogers, P.D., and Fortwendel, J.R. (2020) Mechanisms of triazole

798

799

resistance in Aspergillus fumigatus. Environ Microbiol 22: 4934-4952.

800

O'Gorman, C.M., Fuller, H.T., and Dyer, P.S. (2009) Discovery of a sexual cycle in the

801

opportunistic fungal pathogen Aspergillus fumigatus. Nature 457: 471-474.

802 Pontes, L., Beraquet, C.A.G., Arai, T., Pigolli, G.L., Lyra, L., Watanabe, A., et al. (2020)

803 Aspergillus fumigatus Clinical Isolates Carrying CYP51A with $\mathrm{TR}_{34} / \mathrm{L} 98 \mathrm{H} / \mathrm{S} 297 \mathrm{~T} / \mathrm{F} 495 \mathrm{I}$

804 Substitutions Detected after Four-Year Retrospective Azole Resistance Screening in Brazil.

805 Antimicrob Agents Chemother 64: e02059-19.

806

807 Price, C.L., Parker, J.E., Warrilow, A.G., Kelly, D.E., and Kelly, S.L. (2015) Azole

808 fungicides - understanding resistance mechanisms in agricultural fungal pathogens. Pest Manag

809 Sci71: 1054-1058.

810

811 Qin, W., Liu, C., Jiang, W., Xue, Y., Wang, G., and Liu, S. (20190 A coumarin analogue NFA

812 from endophytic Aspergillus fumigatus improves drought resistance in rice as an antioxidant.

813 BMC Microbiol 19: 50.

814

815 R Core Team (2019). R: A language and environment for statistical computing. R Foundation for

816 Statistical Computing, Vienna, Austria. URL https://www.R-project.org/.

817

818

Resendiz Sharpe, A., Lagrou, K., Meis, J.F., Chowdhary, A., Lockhart, S.R., and Verweij, P.E.

819 (2018) ISHAM/ECMM Aspergillus Resistance Surveillance working group. Triazole resistance

820 surveillance in Aspergillus fumigatus. Med Mycol 56(suppl_1): 83-92.

821

822 Rybak, J.M., Ge, W., Wiederhold, N.P., Parker, J.E., Kelly, S.L., Rogers, P.D., and Fortwendel,

823 J.R. (2019) Mutations in $h m g 1$, Challenging the Paradigm of Clinical Triazole Resistance in

824 Aspergillus fumigatus. mBio 10: e00437-19.

825

826

827

828

Samuel, S., Papayiannis, L.C., Leroch, M., Veloukas, T., Hahn, M., and Karaoglanidis, G.S. (2011) Evaluation of the incidence of the G143A mutation and cytb intron presence in the cytochrome bc-1 gene conferring Qol resistance in Botrytis cinerea populations from several

829 hosts. Pest Manag Sci 67: 1029-1036.

830 
831 Schoustra, S.E., Debets, A.J.M., Rijs, A.J.M.M., Zhang, J., Snelders, E., Leendertse, P.C., et al. 832 (2019) Environmental Hotspots for Azole Resistance Selection of Aspergillus fumigatus, the

833 Netherlands. Emerg Infect Dis 25: 1347-1353.

834

835 Seppey, M., Manni, M., and Zdobnov, E.M. (2019). BUSCO: assessing genome assembly and

836

837

838 annotation completeness. Methods Mol Biol 1962: 227-245.

839

Sewell, T.R., Zhu, J., Rhodes, J., Hagen, F., Meis, J.F., Fisher, M.C., and Jombart, T. (2019)

840

Nonrandom Distribution of Azole Resistance across the Global Population of Aspergillus

841

842

fumigatus. mBio 10: e00392-19.

843

Shen, W., Le, S., Li, Y., and Hu, F. (2016). SeqKit: A cross-platform and ultrafast toolkit for

844

845

FASTA/Q file manipulation. PLOS ONE 11: e0163962.

846

Snelders, E., Camps, S.M., Karawajczyk, A., Schaftenaar, G., Kema, G.H., van der Lee, H.A., et al. (2012) Triazole fungicides can induce cross-resistance to medical triazoles in Aspergillus

847 fumigatus. PLoS One 7: e31801.

848

849

Stamatakis, A. (2014). RAxML version 8: a tool for phylogenetic analysis and post-analysis of

850

851

852

853

854

855

856

857 large phylogenies. Bioinformatics 30: 1312-1313.

858

859 Takahashi-Nakaguchi, A., Muraosa, Y., Hagiwara, D., Sakai, K., Toyotome, T., Watanabe, A., et

860 al. (2015) Genome sequence comparison of Aspergillus fumigatus strains isolated from patients

861 with pulmonary aspergilloma and chronic necrotizing pulmonary aspergillosis. Med Mycol 53:

862 353-360.

863

864 Takahashi, H., Umemura, M., Ninomiya, A., Kusuya, Y., Shimizu, M., Urayama, S., et al. (2021)

865 Interspecies Genomic Variation and Transcriptional Activeness of Secondary

866 Metabolism-Related Genes in Aspergillus Section Fumigati. Front Fungal Biol 2: 656751. doi:

$867 \quad 10.3389 /$ ffunb.2021.656751

868

869 Tenaillon, O., Rodríguez-Verdugo, A., Gaut, R.L., McDonald, P., Bennett, A.F., Long, A.D., et al.

870

871

872 Ter-Hovhannisyan, V., Lomsadze, A., Chernoff, Y. O., and Borodovsky, M. (2008) Gene

873 prediction in novel fungal genomes using an ab initio algorithm with unsupervised training.

874 Genome Res 18: 1979-1990.

875

876 Toyotome, T., Fujiwara, T., Kida, H., Matsumoto, M., Wada, T., and Komatsu, R. (2016) Azole

877 susceptibility in clinical and environmental isolates of Aspergillus fumigatus from eastern

878 Hokkaido, Japan. J Infect Chemother 22: 648-650. 
880 Wang, H.C., Huang, J.C., Lin, Y.H., Chen, Y.H., Hsieh, M.I., Choi, P.C., et al. (2018) Prevalence, 881 mechanisms and genetic relatedness of the human pathogenic fungus Aspergillus fumigatus

882 exhibiting resistance to medical azoles in the environment of Taiwan. Environ Microbiol 20:

883 270-280.

884

885

Yarden, O., and Katan, T. (1993). Mutations leading to substitutions at amino acid 198 and 200

886 of beta-tubulin that correlate with benomyl-resistant phenotypes of field strains of Botrytis

887 cinerea. Phytopathology 83: 1478-1483.

888

889 Zerbino, D.R., and Birney, E. (2008) Velvet: algorithms for de novo short read assembly using de 890 Bruijn graphs. Genome Res 18: 821-829.

891

892

893

Zhang, J., Lopez Jimenez, L., Snelders, E., Debets, A.J.M., Rietveld, A.G., Zwaan, B.J., et al. (2021) Dynamics of Aspergillus fumigatus in Azole Fungicide-Containing Plant Waste in the

894 Netherlands (2016-2017). Appl Environ Microbiol 87: e02295-20.

895

896

Zhang, J., Snelders, E., Zwaan, B.J., Schoustra, S.E., Meis, J.F., van Dijk, K., et al. (2017) A

897 Novel Environmental Azole Resistance Mutation in Aspergillus fumigatus and a Possible Role of

898 Sexual Reproduction in Its Emergence. mBio 8: e00791-17.

899

900

Zhou, D., Korfanty, G.A., Mo, M., Wang, R., Li, X., Li, H., et al. (2021) Extensive Genetic

901 Diversity and Widespread Azole Resistance in Greenhouse Populations of Aspergillus fumigatus

902 in Yunnan, China. mSphere 6: e00066-21.

903

904

Zhou, Z., Duan, Y., and Zhou, M. (2020) Carbendazim-resistance associated $\beta_{2}$-tubulin

905 substitutions increase deoxynivalenol biosynthesis by reducing the interaction between $\beta_{2}$

906 -tubulin and IDH3 in Fusarium graminearum. Environ Microbiol 22: 598-614.

907

908

de Hoon, M.J., Imoto, S., Nolan, J., and Miyano, S. (2004) Open source clustering software.

909

Bioinformatics 20: 1453-1454.

910

911

de Valk, H.A., Meis, J.F., Curfs, I.M., Muehlethaler, K., Mouton, J.W., and Klaassen, C.H. (2005)

912 Use of a novel panel of nine short tandem repeats for exact and high-resolution fingerprinting of

913 Aspergillus fumigatus isolates. J Clin Microbiol 43: 4112-4120.

914

915 van Ingen, J., van der Lee, H.A., Rijs, T.A., Zoll, J., Leenstra, T., Melchers, W.J., and Verweij,

916 P.E. (2015) Azole, polyene and echinocandin MIC distributions for wild-type, $\mathrm{TR}_{34} / \mathrm{L} 98 \mathrm{H}$ and

917 TR46/Y121F/T289A Aspergillus fumigatus isolates in the Netherlands. J Antimicrob Chemother

918 70: 178-181.

919

920 Van Rossum, G., and Drake, F. L. (2009) Python 3 Reference Manual. Scotts Valley, CA:

921 CreateSpace. 
bioRxiv preprint doi: https://doi.org/10.1101/2021.05.23.445375; this version posted May 24, 2021. The copyright holder for this preprint (which was not certified by peer review) is the author/funder, who has granted bioRxiv a license to display the preprint in perpetuity. It is made available under aCC-BY-NC-ND 4.0 International license.

A

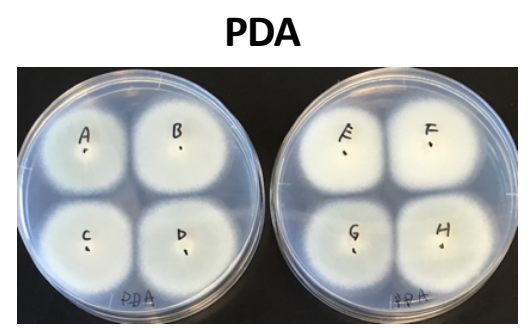

B

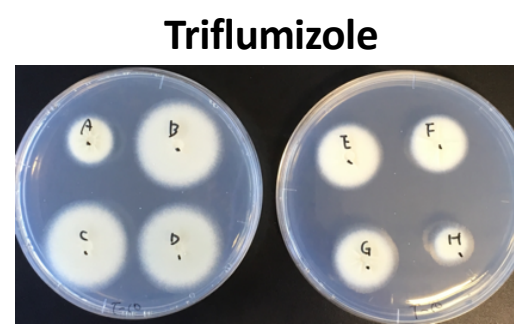

Tebuconazole

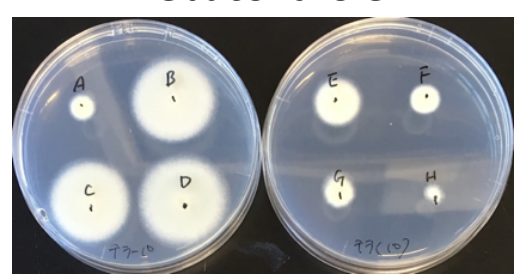

VRCZ

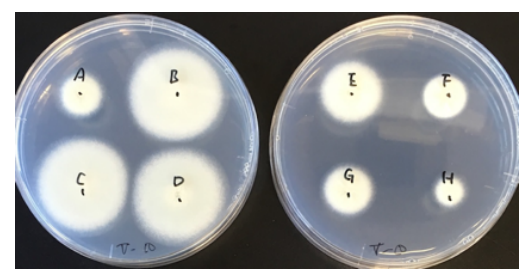

Imazalil

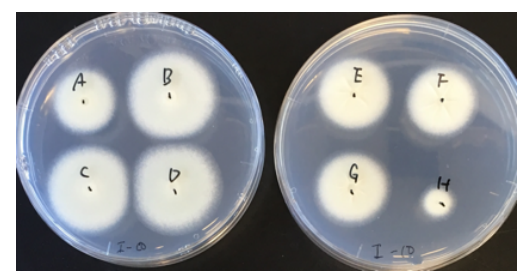

Epoxiconazole

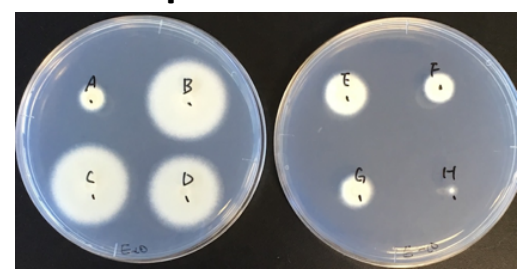

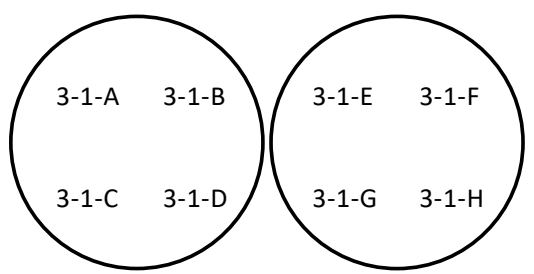

Prochloraz

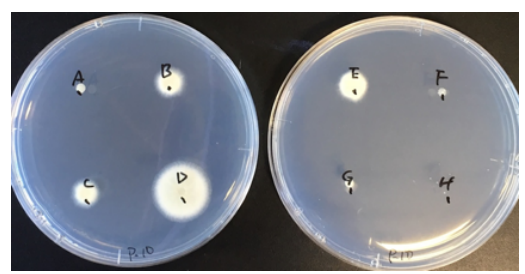

Difenoconazole

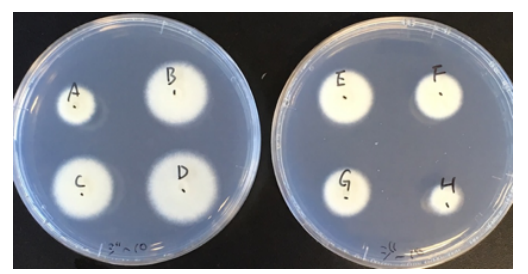

C

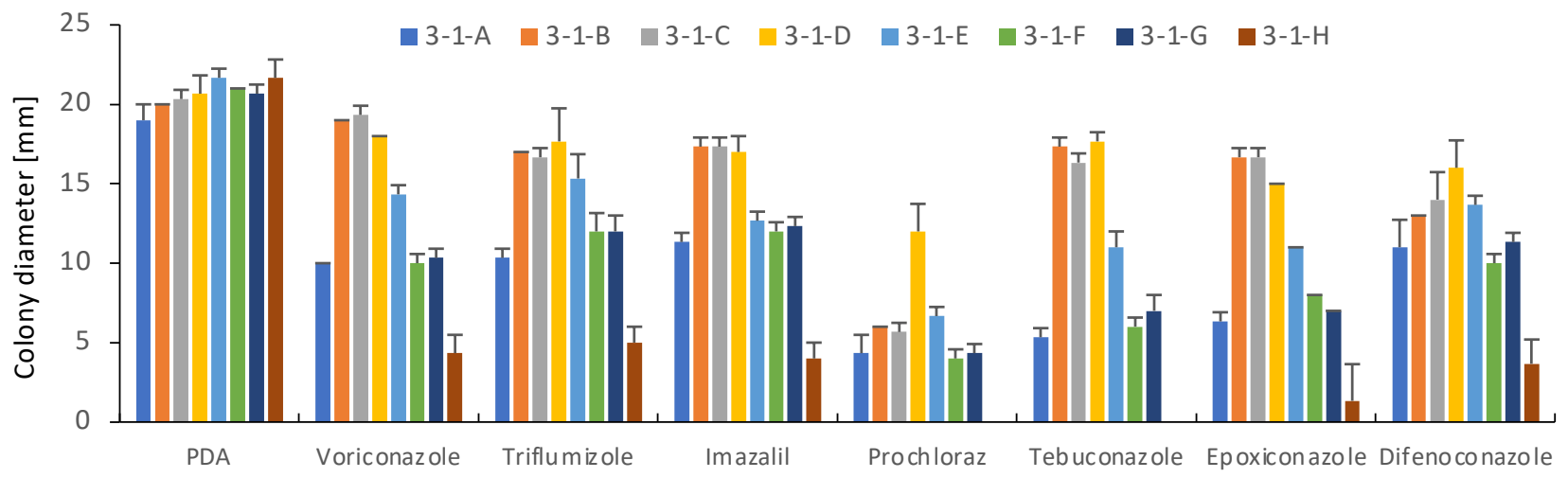

Fig. 1 
bioRxiv preprint doi: https://doi.org/10.1101/2021.05.23.445375; this version posted May 24, 2021. The copyright holder for this preprint (which was not certified by peer review) is the author/funder, who has granted bioRxiv a license to display the preprint in perpetuity. It is made available under aCC-BY-NC-ND 4.0 International license.

A

cyp51A

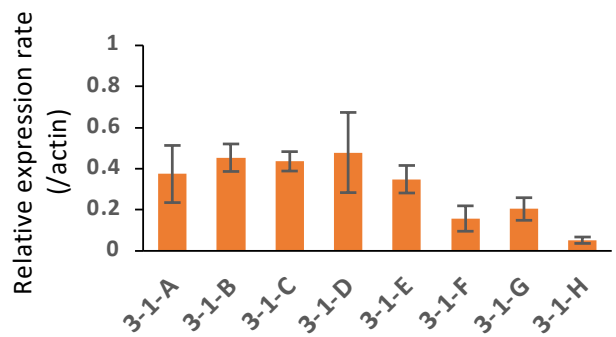

B

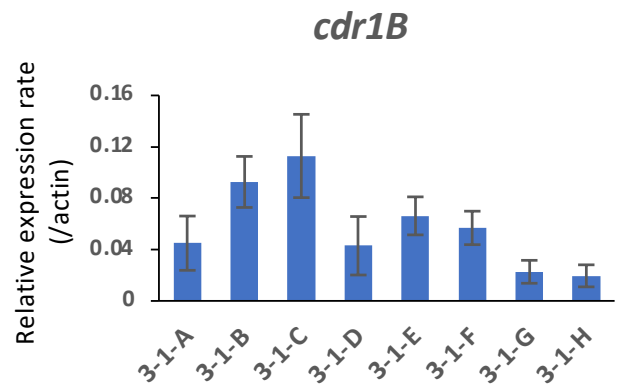


STRAf

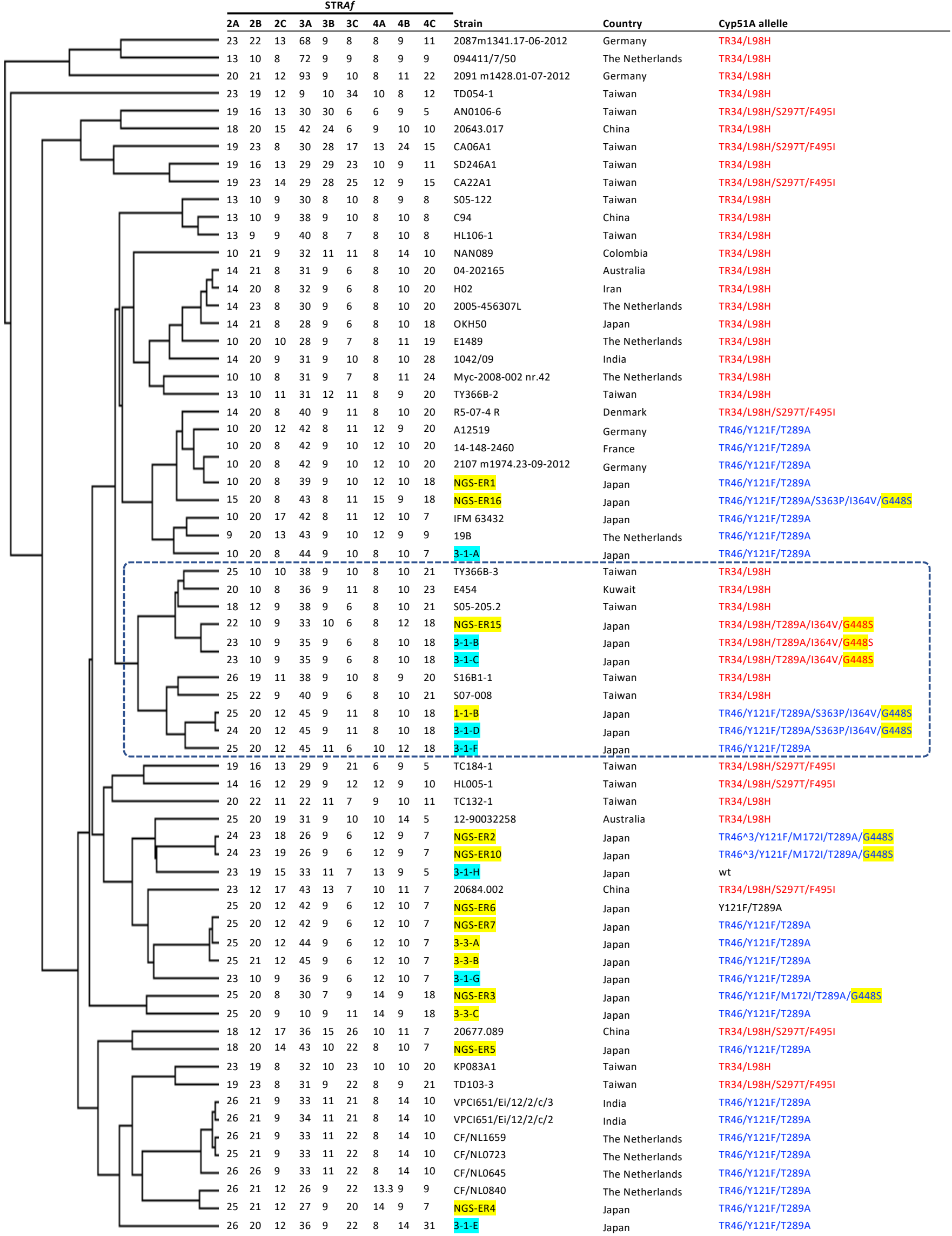

Fig. 3 
A

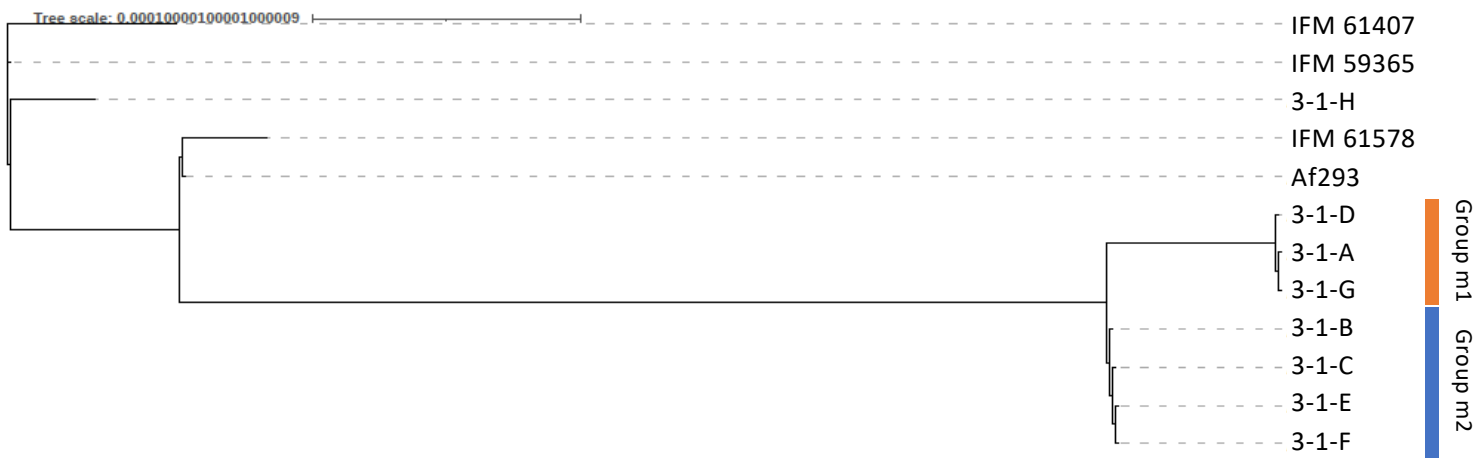

B

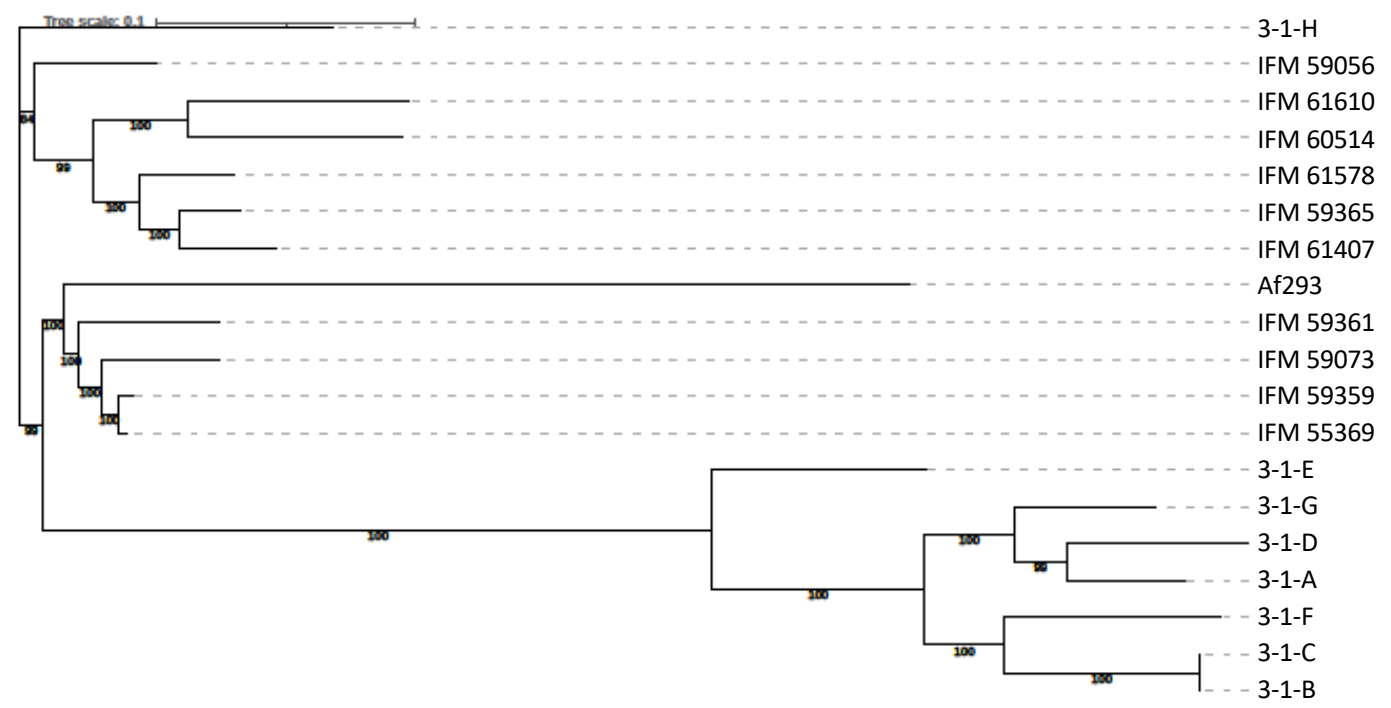

Fig. 4 


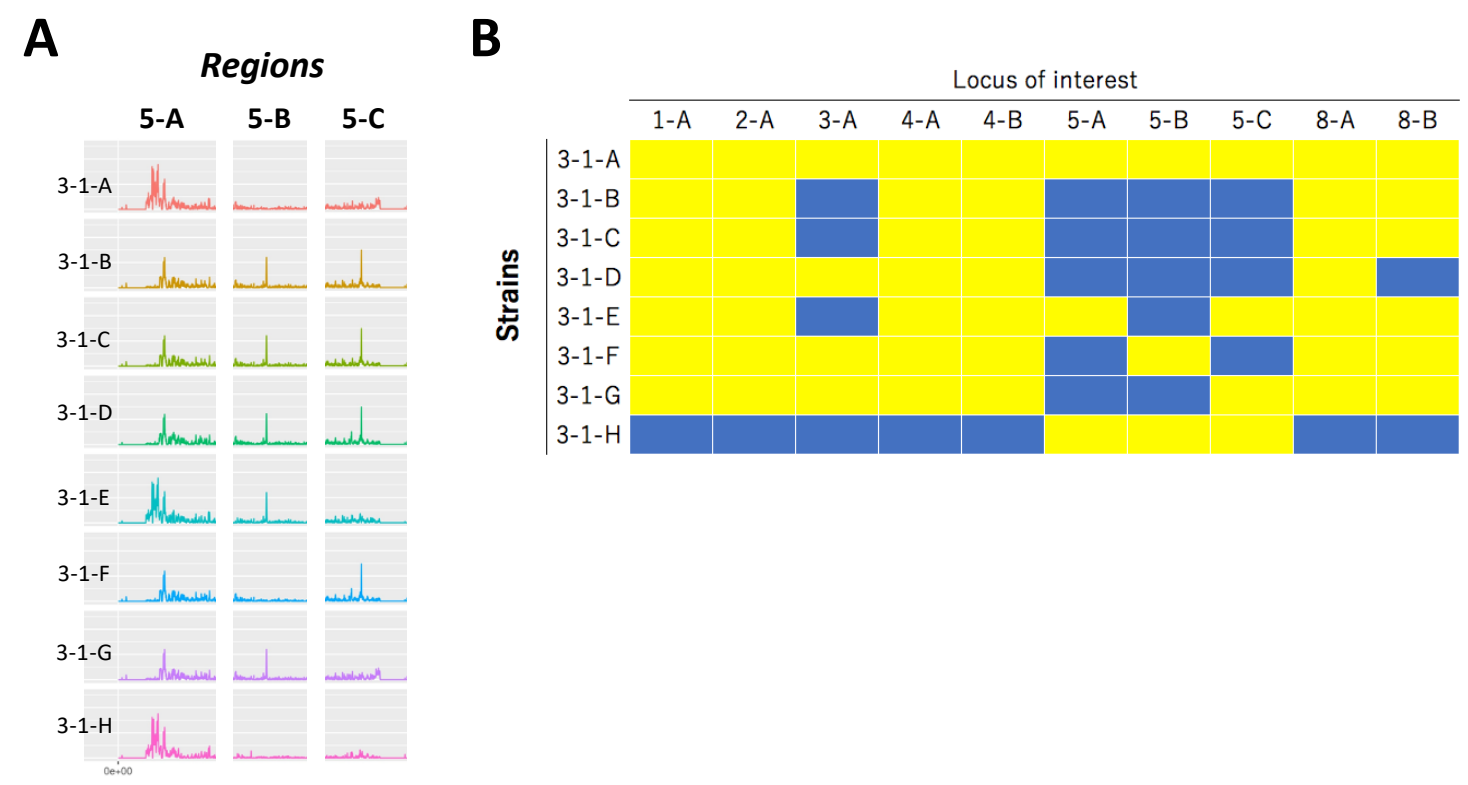

Fig. 5 


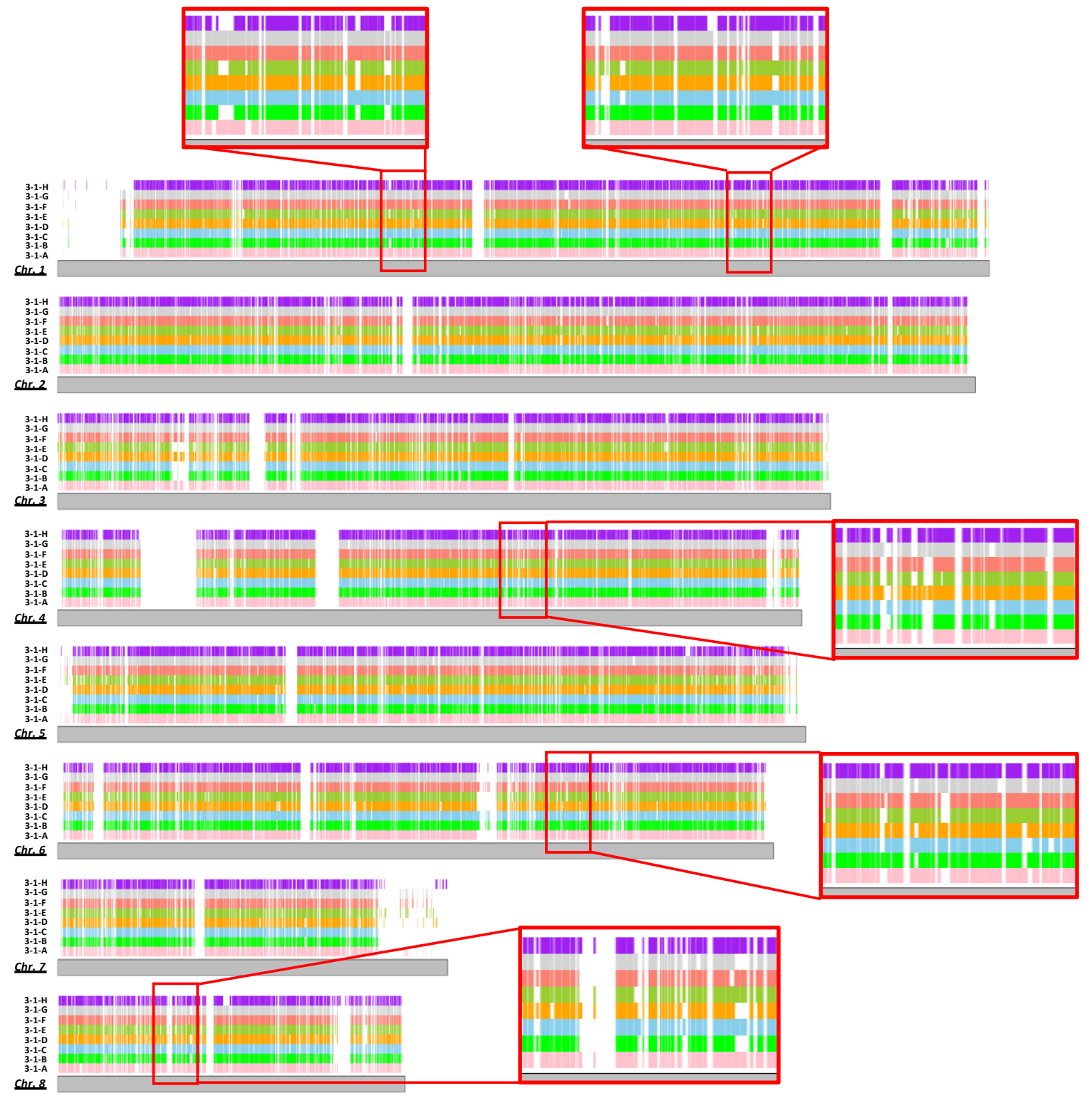

Fig. 6 
A
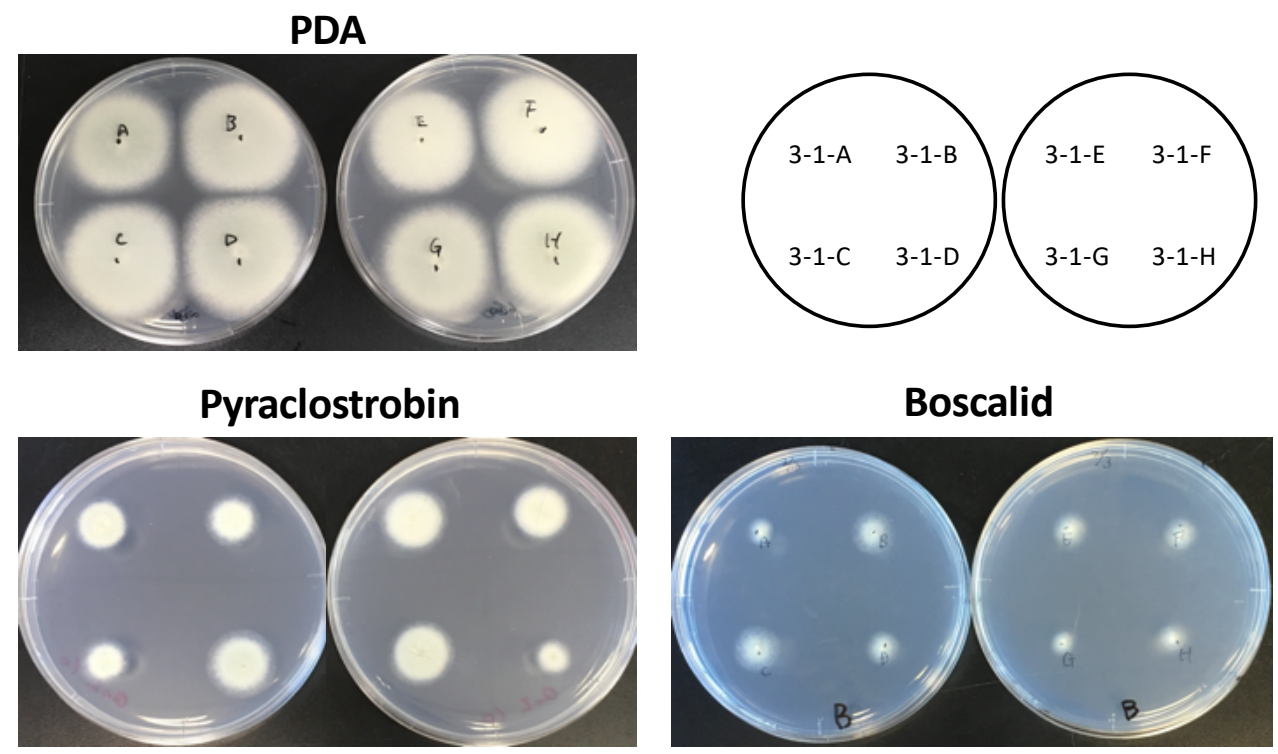

Carbendazim

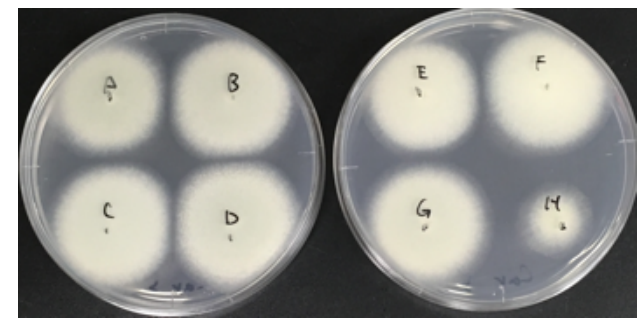

B

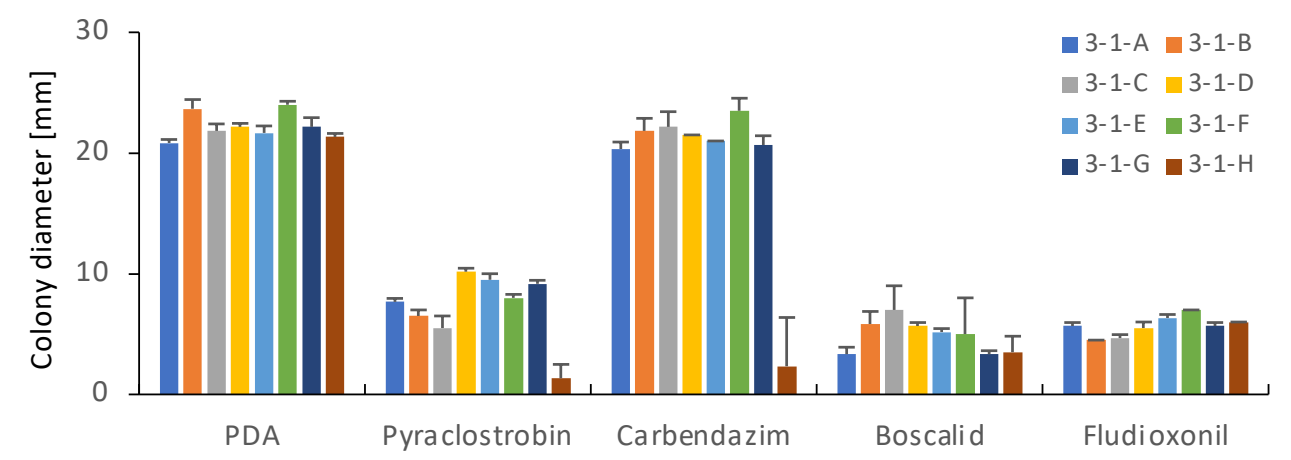

C

CytB (385 aa)

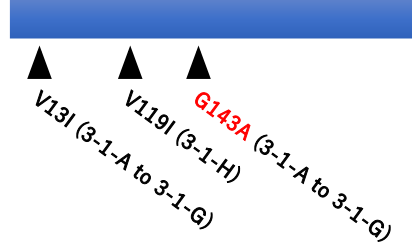

Fludioxonil

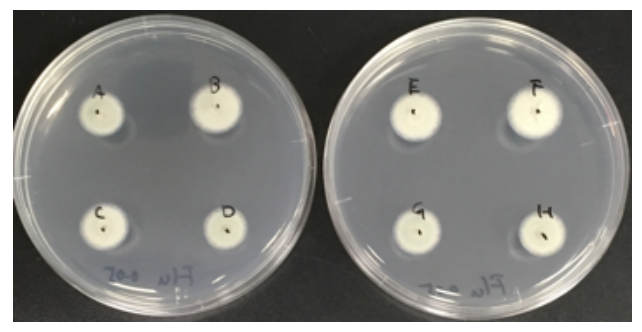

3-1-A $\square 3-1-B$

$C=3-1-$

3-1-G =3-1-H

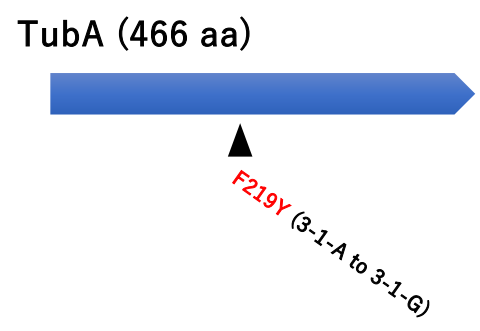

Fig. 7 